\title{
Characterization of the response of quasi-periodic masonry: Geometrical investigation, homogenization and application to the Guimarães castle, Portugal
}

\author{
Gabriele Milani $^{(1)}$, Yhosimi W. Esquivel ${ }^{(2)}$, Paulo B. Lourenço ${ }^{(3)}$, Belén Riveiro $^{(4)}$, Daniel V. \\ Oliveira $^{(5)}$
}

(1) Corresponding Author. Dipartimento di Ingegneria Strutturale (DIS), Politecnico di Milano, Piazza Leonardo da Vinci 32, 20133 Milano, Italy. E-mail: milani@stru.polimi.it

(2) ISISE, University of Minho, Department of Civil Engineering, Azurém, 4800-058 Guimarães, Portugal. yesquivel@pucp.pe

(3) Institute for Sustainability and Innovation in Structural Engineering (ISISE), Dept. of Civil Engineering, School of Engineering, Univ. of Minho, Azurém, 4800-058 Guimarães, Portugal. pbl@civil.uminho.pt.

(4) Department of Materials Engineering. Applied Mechanics \& Construction. University of Vigo, Torrecedeira 86, 36208 Vigo, Spain. belenriveiro@uvigo.es.

(5) Institute for Sustainability and Innovation in Structural Engineering (ISISE), Dept. of Civil Engineering, School of Engineering, Univ. of Minho, Azurém, 4800-058 Guimarães, Portugal. danvco@civil.uminho.pt.

Keywords: Irregular masonry, micro-modeling, homogenization techniques, quasi-periodic masonry, geometrical survey, laser scanning, limit analysis, structural safety. 


\begin{abstract}
In many countries, historical buildings were built with masonry walls constituted by random assemblages of stones of variable dimensions and shapes. The analysis of historic masonry structures requires often complex and expensive computational tools that in many cases are difficult to handle, given this large variability of masonry. The present paper validates a methodology for the characterization of the ultimate response of quasi periodic masonry.

For this purpose, the behaviour at collapse of a wall at the Guimarães castle in Portugal is investigated by means of a rigid-plastic homogenization procedure, accounting for the actual disposition of the blocks constituting the walls and the texture irregularity given by the variability of dimensions in the blocks.

A detailed geometric survey is conducted by means of the laser scanning technique, allowing for a precise characterization of dimensions and disposition of the blocks. After a simplification of the geometry and assuming mortar joints reduced to interfaces, homogenized masonry in- and out-ofplane strength domains are evaluated on a number of different Representing Elements of Volume (RVEs) having different sizes and sampled on the walls of the castle. Strength domains are obtained using a Finite Element (FE) limit analysis approach with a heterogeneous discretization of the RVEs with triangular elements representing units and interfaces (mortar joints), at different orientations of the principal actions with respect to the horizontal direction. The role played by vertical compression is also investigated, considering the case of masonry with weak and strong mortar.

Finally, a series of limit analyses are carried out at structural level, using two different FE numerical models of the so-called Alcaçova wall, a representative perimeter wall of the caste. The first model is built with a heterogeneous material and the second model is built with a homogeneous material obtained through the homogenization procedure performed previously. The purpose is to determinate the reliability of results, in terms of limit load and failure mechanism, for the homogenized model and to compare these results to the ones obtained with the heterogeneous model.
\end{abstract}




\section{Introduction}

Masonry constituted by the assemblage of blocks with variable dimensions is very common in both existing and historical buildings. However, the complexity of the problem, and the number of variables required by accurate numerical heterogeneous FE analyses [1]-[5], usually preclude the study of these structures in the inelastic range through commercial software. As a consequence, it can be stated that, at present, the numerical analysis of masonry structures randomly assembled remains a very challenging problem, despite the efforts recently expended by many authors to tackle the problem using stochastic homogenization schemes [6]-[8].

When dealing with a random assemblage of stones, a key problem is the definition and the use of a suitable material constitutive law at a macro-scale. Homogenization [9]-[15] seems the most straightforward approach, representing a good compromise between micro [16] [17] and macromodelling [18] [19] and allowing the derivation in a rational way of stress-strain relationships for masonry, suitably taking into account texture and mechanical properties of the constituent materials only at a cell level. Additionally, homogenization may lead to the utilization of effective models, with reduced computational effort for real scale simulations [10]-[14].

To corroborate the idea that homogenization is a suitable tool for the analysis of masonry with blocks having variable dimensions, it is noted that most models available in the literature for irregular masonry are based on homogenization concepts (e.g. [20]-[28]). These contributions deal usually with the analysis of irregular masonry within the linear elastic range [20]-[25]. Non-linear analyses through homogenization are relatively common for periodic arrangements (e.g. [9]-[14]), less investigated for random FRP materials [29], and definitely under investigated for quasi periodic masonry and random stones assemblages. Indeed, despite attractive, homogenization remains difficult as it must handle several elementary cells under different loading conditions, with different dimensions and locations in the wall. In this context, the prediction of the load bearing capacity of 
random walls by means of limit analysis is valuable, particularly if it can be managed on standard PCs within a reasonable computation effort [6] [8].

The present paper applies stochastic homogenization concepts for masonry constituted by blocks with variable dimensions, to a real case study of historical interest -the Guimarães castle in Portugal-, assuming that joints are reduced to interfaces and stone blocks have a rigid-plastic behaviour. The hypotheses adopted for mortar and blocks allow combining homogenization of quasi periodic masonry with limit analyses theorems [30]-[33].

Despite the fact that recent and less recent literature (e.g. [9], [31]-[34]) has widely shown that very reliable predictions of collapse loads and failure mechanisms of real scale structures may be obtained with limit analysis, its validity limits must be also considered with care. Among others, a remarkable point concerns the assumption of ductile behavior of the interfaces connecting the stone blocks, which is crucial for the application of limit analysis approaches at the meso- and macroscale. Indeed, the real behavior of the interfaces is quasi-brittle in tension with sudden rupture. At least in principle, one could expect different behaviors between ductile and brittle models. However, such differences tend to be moderate when joint tensile strength is low, as it usually occurs for masonry.

First, a characterization of the masonry geometry under consideration is discussed and a statistical analysis regarding the dimensions of the stones forming the walls of Guimarães castle is carried out. This is followed by a homogenized limit analysis on several Representative Volume Elements (RVEs) from the Alcaçova wall in the Guimarães castle, performed in order to obtain in-plane an out-of-plane failure surfaces to be utilized at a structural level, considering the case of masonry with weak and strong mortar. Afterwards, out-of-plane limit analyses are carried out using two different FE numerical models of Alcaçova wall, being the first one built with a heterogeneous material and the second one with a homogeneous material that was obtained through the homogenization procedure performed in the previous step. The hypotheses adopted for the mortar joints approximate the behaviour for the present condition of the walls and after the planned conservation works. 


\section{Rigid-plastic homogenization in presence of blocks with variable}

\section{dimensions}

The homogenization theory may represent a powerful tool in problems involving periodic arrangements of heterogeneous materials, where the study of the whole structure within a heterogeneous approach is impractical or impossible, due to the computational effort. A nonrigorous homogenization may be also attempted for random composites, provided that a suitable portion of the whole structure (test window) is extracted.

The basic idea of homogenization [10]- [13] consists in introducing averaged quantities representing the macroscopic stress and strain tensors (respectively $\boldsymbol{\Sigma}$ and $\mathbf{E}$ ) as follows:

$$
\begin{aligned}
& \mathbf{E}=<\boldsymbol{\varepsilon}>=\frac{1}{A} \int_{Y} \boldsymbol{\varepsilon}(\mathbf{u}) d Y \\
& \boldsymbol{\Sigma}=<\boldsymbol{\sigma}>=\frac{1}{A} \int_{Y} \boldsymbol{\sigma} d Y,
\end{aligned}
$$

where $A$ stands for the area of the elementary cell, $\boldsymbol{\varepsilon}$ and $\boldsymbol{\sigma}$ stand for the local quantities (stresses and strains respectively) and $<*>$ is the averaging operator.

Periodicity conditions are imposed on the stress field $\boldsymbol{\sigma}$ and the displacement field $\mathbf{u}$, given by:

$\left\{\begin{array}{l}\mathbf{u}=\mathbf{E y}+\mathbf{u}^{\text {per }} \quad \mathbf{u}^{\text {per }} \text { on } \partial Y \\ \boldsymbol{\sigma} \mathbf{n} \text { anti - periodic on } \partial Y\end{array}\right.$

where $\mathbf{u}$ is the total displacement field and $\mathbf{u}^{\text {per }}$ stands for a periodic displacement. Here, it is worth noting that, in equation ( 2 ), the periodicity of the displacement fluctuation $\mathbf{u}^{\text {per }}$ forces corresponding boundary segments to exhibit the same shape in the deformed configuration. For instance, within a displacement based FE code, a linear constraint relation between couples of nodes may be imposed. Let us consider two points, say $\mathrm{P}_{1}$ and $\mathrm{P}_{2}$ on opposite boundary sides of the RVE, see Figure 1, such that $y_{1}\left(\mathrm{P}_{1}\right)=y_{1}\left(\mathrm{P}_{2}\right)+L$ and $y_{2}\left(\mathrm{P}_{1}\right)=y_{2}\left(\mathrm{P}_{2}\right)$ where $y_{1}-y_{2}$ indicate a local frame of reference with origin placed on the center of the RVE and $L$ denotes RVE size along $y_{1}$. Within 
such assumptions, the linear constraint relation reads $\mathbf{u}_{P 2}=\mathbf{u}_{\mathrm{P} 1}^{\mathrm{per}}+\mathbf{E}\left(\mathrm{P}_{2}-\mathrm{P}_{1}\right)$, with $\mathbf{u}_{\mathrm{P} 1}^{\mathrm{per}}=\mathbf{u}_{\mathrm{P} 2}^{\mathrm{per}}$. The macroscopic homogenized quantity $\mathbf{E}$ may then be imposed on the cell through boundary nodes displacements. Conversely, anti-periodicity of the stress field requires that the micro-stress vector $\boldsymbol{\sigma n}$ is mirrored on opposite sides of $\partial Y$, as depicted in Figure 1.

Let $S^{\mathrm{m}}, S^{\mathrm{b}}$ and $S^{\text {hom }}$ denote respectively the strength domains of mortar, blocks and homogenised macroscopic material. It has been shown by Suquet [30] that a kinematic upper bound approximation of $S^{\text {hom }}$ can be derived through the support function $\pi^{\text {hom }}(\mathbf{D})$ as follows:

$$
S^{\text {hom }}=\left\{\sum \mid\left\{\begin{array}{c}
\sum: \mathbf{D} \leq \pi^{\text {hom }}(\mathbf{D}) \quad \forall \mathbf{D} \in R^{6} \\
\pi^{\text {hom }}(\mathbf{D})=\inf _{\mathbf{v}}\left\{P(\mathbf{v}) \mid \mathbf{D}=\frac{1}{2 \Gamma} \int_{\partial Y=\Gamma}(\mathbf{v} \otimes \mathbf{n}+\mathbf{n} \otimes \mathbf{v}) d S\right\} \\
P(\mathbf{v})=\int_{Y} \pi(\mathbf{d}) d Y+\int_{S} \pi([[\mathbf{v}]] ; \mathbf{n}) d S
\end{array}\right\}\right.
$$

where:

- $\quad \mathbf{v}=\mathbf{D y}+\mathbf{v}^{\text {per }}$ is the microscopic velocity field;

- $\quad \mathbf{v}^{\text {per }}$ is a periodic velocity field;

- $\quad \mathbf{d}$ and $\mathbf{D}$ are respectively the microscopic and macroscopic strain rate fields;

- $S$ is any discontinuity surface of $v$ in $Y, \mathbf{n}$ is the normal to $S$;

$-\quad \pi([[\mathbf{v}]] ; \mathbf{n})=1 / 2([[\mathbf{v}]] \otimes \mathbf{n}+\mathbf{n} \otimes[[\mathbf{v}]]) ;$

$-\pi(\mathbf{d})=\max _{\boldsymbol{\sigma}}\{\boldsymbol{\sigma}: \mathbf{d} ; \boldsymbol{\sigma} \in S(\mathbf{y})\}$.

The above considerations hold on a Representative Element of Volume (RVE) that should generate the entire wall by repetition. The RVE is defined as the smallest volume that contains all the essential information about the microstructure. On the boundaries of the RVE, periodicity and antiperiodicity conditions should be assigned in agreement with equation ( 2 ).

However, the identification of a RVE for old masonry is not always an easy task, as is the case here analysed. Indeed, using adequate translations for a periodic structure, a unit cell that generates the 
whole structure can be used [10]. In composite material such as fibre-reinforced ones, it is sufficient to choose a relatively large portion of material, depending on the size of the inclusions, to determine the RVE. The definition of the material statistical homogeneity and the proof of convergence when the material sample size approaches infinity are addressed in [35]-[37]. For the problem at hand, it should be noted that the inclusions (irregular stones) in the matrix (the mortar) can assume different relative dimensions and arrangement, depending on the portion of masonry analysed.

The method here developed to find an approximation of macroscopic masonry ultimate strength is an iterative one and follows the procedure envisaged in [20], assuming rigid plastic behaviour of the constituent materials. The following steps are necessary to obtain macroscopic strength domains:

1. The first phase consists in choosing a rectangle, the so called test-window, which contains a sufficient portion of both phases (irregular stones and mortar) in an entire wall. Since its position and dimension are fully arbitrary, ideally, a large Monte Carlo sampling of different test windows (differing in both dimension and position) should be performed, to handle statistically the output resultant masonry macroscopic strength. Obviously, the larger is the number of windows considered, the harder is the computational effort required to have an estimation of the macroscopic resistance. As a rule, the utilization of small test windows should be withdrawn. Heuristic rules suggest to avoid sizes smaller that 3-4 times the maximum dimensions of the stones [27]. Within a FE discretization of the test window, this compromise leads to medium size problems. In what follows, in order to obtain a relatively fast estimation of masonry strength domain, several different test windows differing in size have been considered.

2. The next phase is to determine the strength values of the test window in plane stress conditions, see for instance [28]. This information is retrieved discretizing the test window into a triangular heterogeneous mesh and in solving, by means of a plane stress limit analysis code, a standard homogenization problem in the rigid plastic case (upper-bound 
solution). All the numerical models used here for limit analysis belong to the upper bound family and are based on the kinematic discontinuous formulation originally presented by Sloan and Kleeman [38] and applied many times for masonry (e.g. [39][40]). Theoretical details of the code are given in Appendix A.

3. The final phase is to implement the mean homogenized strength domains at structural level, using a homogeneous failure surface. To perform the numerical simulations at structural level, the mean failure surface previously evaluated is adopted, in agreement with [6] [8].

\section{Geometric characterization of the Guimarães castle}

The origin of the castle dates back to the $10^{\text {th }}$ century and the fights against the Moors in the Iberian Peninsula. In the $11^{\text {th }}$ century, the first King of Portugal was born there. Later, between the $12^{\text {th }}$ and $14^{\text {th }}$ centuries, the castle was enlarged and the defence capacity was improved. At a certain stage, the castle was abandoned and suffered damage caused by time, and by the subsequent changes of use. In the $20^{\text {th }}$ century, important restoration works have been carried out and the current condition is shown in Figure 2. The castle is surrounded by eight square towers, which delimit the main square, with a main tower ("Torre de Menagem" in Portuguese and "Keep" in English) in the centre. The main wall under study in this paper is the so-called "Alcáçova” Wall, which is originally the highest and most protected part of an Iberian medieval castle, with a defence function and where the civil or ecclesiastical authorities lived. The word was later used to define the part of the castle where the governor lived.

The plan view of the castle is relatively simple, with a pentagonal (Figure 2-b) shape in plan. The castle is also surrounded by eight square towers, delimiting the main geometry, and protecting the main tower. The current version of the castle has Romanesque origin, with a geometry resembling a Middle Age shield, with a small central chamber and difficult access. 


\subsection{Automatic geometry extraction of quasi-periodic masonry walls by terrestrial laser scanning}

The masonry material characterizing the geometry of the castle is constituted by granite stone ashlars in the external leaves. Horizontal mortar courses are relatively regular, despite the fact that the height of the courses is not constant and that the length of the units is rather variable (see for instance Figure 2-c). In order to represent this feature, a statistical description considering mean, standard deviation, coefficient of variation and probability distribution for the size of the stones on four walls is investigated. The statistical study was possible thanks to a detailed laser scanning survey conducted on the whole structure, which allowed determining the size of each stone automatically.

Laser scanning was used in order to have an extensive and accurate geometric documentation of the castle. Data processing usually involves a tedious processing and managing such massive data requires advanced knowledge in geomatics. Under the aim of having a tool that allows accurate geometric documentation, avoiding deep skills in laser scanning processing, an algorithm for automatic geometry extraction of quasi-periodic masonry walls was developed using Matlab software. The results obtained in some wall samples of Guimarães castle were compared with a simpler photography based procedure in order to validate, in terms of quality, the usage of the second method when access to a laser scanning instruments is not possible. Next, the main steps of the methodology are summarized.

\subsection{Data acquisition and pre-processing}

A terrestrial laser scanner model RIEGL LMS Z-390i was used to collect the geometry of the external walls of the Castle. This laser scanner is able to record long distances (up to $400 \mathrm{~m}$ in normal light conditions) with a nominal accuracy of $6 \mathrm{~mm}$ at $50 \mathrm{~m}$. The technical specifications of the equipment are given in [41]. The acquisition rate reaches values of 11,000 points per second 
which allowed collecting the geometry of the tower walls used for the comparison very fast (point clouds bigger that 6 million points took about 20 minutes). The spatial resolution of the point clouds was set to 0.02 degrees ( $3 \mathrm{~mm}$ at $10 \mathrm{~m}$ range) for both horizontal and vertical angles. The raw point cloud is collected into a central coordinate system, where each point position is defined by spherical coordinates: a distance and two angles (vertical, referred to the zenith of the instrument; and horizontal, referred to its azimuth). The instrument is managed using the software Riscan Pro, where the raw data is stored. Points are automatically converted to a Cartesian coordinate system (XYZ).

In addition, most laser scanners have the ability of recording the "intensity" data. This parameter refers to an attribute that represents the amount of energy of the returned pulse, see Figure 3. This intensity is expressed as a percentage of the emitted laser pulse [42] and is considered to be a key attribute to perform classification of ashlars and joints (with or without mortar) in masonry walls. From the results of the segmentation, morphology analysis of ashlars can be done. Once the walls are scanned, smaller point clouds are used as sample walls to serve as input data for the algorithm developed. These point clouds are exported to ASCII format containing the Cartesian coordinates and intensity attribute for each point.

The algorithm was designed to perform point cloud data segmentation based on digital image processing using the so-called 2.5D approach. Due to the overall geometric characteristics of masonry walls, normally forming planar surfaces as a whole, a planar projection of the 3D point cloud is performed. To achieve this, wall planes are estimated performing an orthogonal planar fitting of the whole sample wall. After obtaining a plane for projection, all the 3D points are orthogonally projected over the fitted plane. The next step consists of transforming the vector planar image to a raster image. For that process, it is essential to fix a cell size that will correspond with the spatial resolution of the processing data. Taking into account the resolution of the original point cloud, a square cell of $1 \mathrm{~cm}$-width and $1 \mathrm{~cm}$-height was fixed for the images. The digital number of 
each cell is computed as the averaged intensity value of the points contained, so the intensity image of the wall is obtained.

Figure 4 shows intensity images of the walls from Guimarães castle. Once an image is created, the new task consists of the definition of each individual block in order to obtain its dimensions (length and height). Also, the user may select a smaller window of segmentation into the whole image in order to facilitate the statistical random sampling. The dimensional analysis for each sample window is subsequently computed. The core principle used for ashlar segmentation is based on an edge detection algorithm using the so-called Sobel operator [38]. By computing directional intensity gradients (first derivative of intensity image) it is possible to detect abrupt differences between each pixel and its neighbours. After performing directional projections of the previous functions, joints are accurately detected and block dimensions can be stored. Figure 5 summarizes the process of joint detection and geometric analysis of masonry blocks. From this methodology it was possible to obtain the length and height of masonry blocks composing the walls of Guimarães castle using a long-range terrestrial laser scanner. The final accuracy of the laser scanning processing algorithm resulted to be between 1-2cm (pixel size in intensity image), depending on the accessibility conditions to the sample walls. Table 1 summarizes morphological analysis of two sample walls, and shows a comparison between the photographic method and the laser scan. It can be observed that a reasonable agreement is found, even if the sample is not exactly the same.

For the sake of clarity, the four walls chosen for such a detailed study will be denoted hereafter as Wall 1, Wall 2, Tower Wall and Alcaçova Wall. Their location is clearly identified in Figure 2-b and the photographic survey was used, as the laser scan results and edge extraction process was only recently completed.

The statistical analysis was carried out as follows: firstly, each wall was considered independently, evaluating the statistical distribution of the length and the height of the stones; then, all data were considered together, in order to evaluate the actual overall distributions on the entire castle. 
The numbers of stones considered for each sample were the following: Wall 1 has 110 units, Wall 2 110 units, Tower wall 110 units and Alcaçova wall 308 units. The results from the statistical analysis of Wall 1, Wall 2, Tower wall, Alcaçova wall and the entire sample walls are presented from Figure 6 to Figure 9. In particular, in Figure 6, the statistical distributions obtained for the length and the height of the stones belonging to Wall 1 are depicted. A best fit, assuming 3parameters lognormal probabilistic distributions for both variables is also represented. The mean values for the length and height of the stones are $75.8 \mathrm{~cm}$ and $38.9 \mathrm{~cm}$ respectively. The stones' length is typically around $67.5 \mathrm{~cm}$ and the height of the stones is typically about $40 \mathrm{~cm}$.

When dealing with Wall 2 (see Figure 7), the mean values of the length and height are $69.7 \mathrm{~cm}$ and $43.8 \mathrm{~cm}$, respectively. The length of the stones is typically about $65 \mathrm{~cm}$ and the height of the stones is around $40 \mathrm{~cm}$. The best fitting probabilistic distribution for both variables is sketched in Figure 7. When dealing with the Tower wall (Figure 8), the mean values for stones length and height are 85.2 $\mathrm{cm}$ and $45.7 \mathrm{~cm}$, respectively, and the height of the stones is typically about $49.5 \mathrm{~cm}$. The probabilistic distribution for both variables is depicted in Figure 8. In the Alcaçova wall (see Figure 9), the mean values for the length and the height of the stones are $59.8 \mathrm{~cm}$ and $33.5 \mathrm{~cm}$, respectively, and the typical height of the stones is about $39 \mathrm{~cm}$.

When all samples are considered together, length and height mean values are equal to $68.6 \mathrm{~cm}$ and 38.4 respectively, with standard deviations equal to $27.8 \mathrm{~cm}(\mathrm{CoV}$ of $40 \%)$ and $9.26 \mathrm{~cm}(\mathrm{CoV}$ of 24\%). The typical length of the stones is about $55 \mathrm{~cm}$. Lengths between $50 \mathrm{~cm}$ and $60 \mathrm{~cm}$ have the largest frequency (133 on 639 stones). A second group of stones with a large frequency exists (125), with lengths ranging between 60 and $70 \mathrm{~cm}$. The smallest length is about $25 \mathrm{~cm}$ and the $210 \mathrm{~cm}$, being both atypical. The histogram is slightly skewed to the right. The typical stones height is about $39 \mathrm{~cm}$. Heights between $38 \mathrm{~cm}$ and $40 \mathrm{~cm}$ are quite frequent. The smallest height is $13 \mathrm{~cm}$ and the largest about $62 \mathrm{~cm}$, which are both very atypical. The probabilistic distributions for both variables using a 3-parameters lognormal model are summarized in Figure 10. 
A summary of the geometrical data is reported in Table 2. From the statistical analysis conducted, the following major issues are worth noting:

- A large variability of the mean length and height values of the stones for the four walls analysed is observed (from 60 to $80 \mathrm{~cm}$ in length and from 34 to $46 \mathrm{~cm}$ in height). The ratio between the maximum and minimum value found is similar for both quantities (about 75\%).

- The stones geometrical ratio between height and length $(\mathrm{h} / \mathrm{b})$ is rather important for the quality of the masonry bond. $\mathrm{h} / \mathrm{b}$ average ratio is about $56 \%$ (1:1.8). Only in Wall 2, a slightly different $\mathrm{h} / \mathrm{b}$ ratio is found, equal to $63 \%(1: 1.6)$.

- The scatter found on length values is always much larger than that found on height values, being the scatter of the full sample not so much different from the scatter of each individual sample.

- Wall 2 is the sample with lowest scatter, whereas the Alcaçova wall is the sample with the largest scatter, despite that fact that the Alcaçova number of stones is three times larger than Wall 2.

- The difference found between an averaging procedure performed on the total sample weighting the number of samples or obtained assuming the same weight is moderate, with a change in the dimensions found near $5 \%$.

- The probabilistic length distributions are clearly skewed, requiring for fitting a lognormal distribution. The probabilistic height distribution is relatively symmetric, meaning that a normal distribution can be used.

\section{FE homogenized failure surfaces: Numerical results}

To fully characterize the macroscopic masonry behaviour at failure when a plane stress condition is assumed, it is necessary to provide a strength domain in the space of membrane actions $\Sigma_{11}$ 
(horizontal homogenized normal stress), $\Sigma_{22}$ (vertical homogenized normal stress), $\Sigma_{12}$ (shear homogenized stress).

Traditionally, a 2D representation of such surfaces is given by many authors [6] [8] [31] providing three different 2D sections of the 3D surface at three fixed angles $\vartheta$, defined as the angle between the horizontal direction and the homogenized principal stress $\sigma_{h h}$. Assuming $\sigma_{v v}$ the principal stress acting orthogonally to $\sigma_{h h}$, such sections are obtained keeping $\vartheta$ fixed and varying point by point a further angle, say $\psi$, defined as $\psi=\tan ^{-1} \sigma_{v v} / \sigma_{h h}$. Within this assumption, the homogenized membrane resistances $\Sigma_{11}, \Sigma_{22}$ and $\Sigma_{12}$ are defined as follows:

$$
\begin{aligned}
& \Sigma_{11}=\frac{\lambda}{2}(\cos (\psi)(1+\cos (2 \vartheta))+\sin (\psi)(1-\cos (2 \vartheta))) \\
& \Sigma_{22}=\frac{\lambda}{2}(\cos (\psi)(1-\cos (2 \vartheta))+\sin (\psi)(1+\cos (2 \vartheta))) \\
& \Sigma_{12}=\frac{\lambda}{2}(\cos (\psi) \cos (2 \vartheta)-\sin (\psi) \cos (2 \vartheta)) \tan (2 \vartheta)
\end{aligned}
$$

where $\lambda$ is the load multiplier.

The FE models representing the RVEs are built using different samples of walls from the Alcaçova wall with different sizes. The wall is built using two external leaves with an average thickness of $400 \mathrm{~mm}$ and separated by an infill.

Here it is worth mentioning that out-of-plane homogenized strength domains reported in what follows refer to a single external leaf with thickness equal to $400 \mathrm{~mm}$. Since both infill mechanical properties are very poor (cohesion in practice vanishing and friction angle equal to $20^{\circ}$ ) and its thickness is relatively small (200 mm), a conservative hypothesis has been adopted disregarding the effect induced by the interlocking between external leafs and infill. Thus, the overall ultimate moments of the façade have been evaluated as the sum of the contributions of the single leafs, assuming for the infill an isotropic behaviour obeying a Mohr-Coulomb failure criterion (ultimate bending moment and torsion may be easily evaluated by means of trivial formulas). The hypothesis that leafs bend separately has therefore implicitly made. Finally, it has to be noted that the 
subdivision of each leaf with 12 layers provides quite good estimates of the out-of-plane failure loads, as shown in [9].

The wall has 308 stones irregularly disposed, but maintaining the horizontal alignment of the bed joints (coursed masonry). It was decided to consider three RVEs of different size, labelled as 3x3, $4 \times 4$ and 5x5, having dimensions respectively equal to three, four and five times the mean length and the mean height of the stones. For each RVE size, three different samples located randomly on the wall are taken into account (Figure 11). In addition, three artificial RVEs are built using mean size stones and periodic arrangement, in order to compare the resulting failure surfaces with those obtained considering the actual geometry and disposition of the blocks.

It is worth noting that the so called random test windows method is utilized to have an estimate of the masonry behavior at the macro-scale. The stochastic analysis on wall texture and conducted in the previous section is done to have an insight into average values of blocks height and length and build the artificial RVEs with comparison purposes. A fully stochastic approach is not adopted in the paper because it would require large scale Monte Carlo simulations, basing on stochastic distributions found from the survey, as envisaged in [8]. Large scale numerical computations on the case study considered are without the aim of the present research, which remains limited to the proposal of a method for a rough evaluation of collapse loads useful from an engineering viewpoint. A certain scatter on predicted homogenized properties is obviously possible when using the test window method, as demonstrated in another context by Zeman and Sejnoha [29], depending on the size of the test window (large test windows are linked to data at failure exhibiting less scatter) and the actual local disposition of the bricks. Since for the case analyzed, the length of the blocks and their disposition vary with sufficient regularity, the scatter obtained is in any case rather limited.

A linearized Lourenço and Rots [2] failure criterion is adopted for joints reduced to interfaces whereas a classic Mohr-Coulomb failure criterion is used for block-block interfaces (see [3] for details). Triangular rigid elements are utilized to mesh the RVEs, in order to speed up calculations. Despite the fact that plastic dissipation is allowed in the model only on interfaces, this does not 
represent either a limitation or a lack of accuracy of the numerical model. It is expected, indeed, that failure occurs for the formation of cracks zigzagging along mortar joints, being the stone strength rather high.

The RVEs considered are depicted in Figure 12 (actual disposition of the stones) and Figure 13 (artificial models), along with their FE discretization (each colour represents a different stone). The main geometrical properties of the RVEs are summarized in Table 3 for the sake of clearness. Furthermore, in Table 4 the number of elements and nodes utilized for the FE discretization is summarized. For each RVE and in each orientation $\vartheta$, 32 values with steps of half of $22.5^{\circ}$ have been calculated. The 32 points were then connected to draw failure surfaces. The optimization problem arising in order to obtain the failure surface is solved by using an algorithm code developed in [31].

Two different cases are considered, assuming a masonry material with good and poor mechanical properties. For masonry with weak mortar, the compressive strength $f_{c}$ of joints reduced to interfaces is assumed equal to $12 \mathrm{MPa}$, with ultimate tensile strength $f_{t}$ equal to $0.05 \mathrm{MPa}$, cohesion c equal to $\mathrm{f}_{t}$, friction angle $\Phi=30^{\circ}$ and shape of the linearized compressed cap expressed by the angle $\Phi_{2}$ equal to $45^{\circ}$. The compressive strength of stones is assumed equal to 89.5 $\mathrm{MPa}$ and their ultimate tensile strength is equal to $0.93 \mathrm{MPa}$, according to experimental characterization reported in [44]. For masonry with strong mortar, only the ultimate tensile strength of masonry is changed, assuming a value equal to $0.3 \mathrm{MPa}$, keeping c equal to $\mathrm{f}_{t}$.

Figure 14 shows some typical in-plane homogenized failure surface sections for a 4x4 RVE in case of weak (-a) and strong (-b) mortar, at different orientations of the principal axes with respect to the bed joint direction. The RVE exhibits the expected anisotropic behaviour.

In Figure 15, a 3D representation of the strength domain in the tension-tension (-a) and compression-compression regions for a $4 \times 4 \mathrm{RVE}$ is provided for the sake of clearness. The typical 
orthotropic behaviour in tension due to blocks staggering is worth noting, as well as a moderate difference between vertical and horizontal compression failure loads is noted.

In Figure 16, a comparison between in-plane homogenized failure surfaces obtained by means of the $4 \times 4$ RVEs and the artificial 4x4 RVE, at different orientations of the principal stress axes with respect to bed joint direction is summarized (-a: weak mortar joint, -b: strong mortar). As can be seen, failure surfaces obtained from real RVEs of the same size exhibit a similar behaviour at failure, while more remarkable deviation is found for the artificial RVE, with a significant difference in horizontal strength. Such a response has been experienced also for 3x3 and 5x5 RVEs. Consequently, from results reported in Figure 16, it can be stated that particular care should be used by the user when artificial RVEs are adopted to have an estimation of the homogenized failure surfaces of masonry with blocks having variable dimensions. While the approach based on artificial RVEs undoubtedly allows saving time and may be easily automated, it could, at least in principle, provide unreliable collapse loads when failure occurs for the activation of yield lines associated to a predicted masonry strength different from the real one.

Furthermore, it is worth mentioning that in both cases of weak and strong mortar, the continuous alignment of the bed joint results in a no variation of the vertical strength $\left(\sigma_{h}\right)$, because the RVEs tend always to fail along a preferential plane of weakness formed by the bed joint.

A large amount of numerical results is obtained, not reported here for the sake of conciseness.

In Figure 17, Figure 18 and Figure 19, a comparison between the mean strength values obtained post-processing results collected for each single RVE is reported respectively for orientations $\vartheta=0^{\circ}$, $22.5^{\circ}$ and $45^{\circ}$ of the principal stress axis with respect to bed joint orientation. The agreement among results obtained with different sizes of the RVEs is very promising, indicating that even a small sampled RVE may provide reasonable estimates of masonry strength.

Some meaningful failure modes obtained from selected RVEs for orientations $\vartheta=0^{\circ}, 22.5^{\circ}$ and $45^{\circ}$ are depicted in Figure 19, where a qualitative comparison with experimental results by Dhanasekar et al. [45] is also shown. A staircase crack in the $4 \times 4$ representative volume element is 
found independently of the quality of the mortar. It is noted that dilatancy is present in the numerical model, even if it is believed that the influence in the global behaviour is very low (the upper boundary is allowed to move up, meaning that an artificial confining stress built up does not occur).

\section{Out-of-plane homogenized failure surfaces}

In order to speed up limit analysis computations, the simplified homogenization approach proposed for the first time in [46] is utilized to obtain out-of-plane homogenized strength domains.

The utilization of alternative procedures is certainly possible, as for instance the utilization of the out-of-plane upper bound code proposed in [8]. However, it was decided to firstly evaluate in-plane homogenized failure surfaces and discuss differences for in-plane actions there, where the comparison is much more straightforward. Then, the layered lower bound model proposed in [46] is utilized to evaluate out-of-plane failure surfaces. It has been shown that reliable predictions of the actual failure surfaces are obtained with [46] model, see for instance [9], with a slight increase of the overall numerical efficiency of the multi-step procedure proposed, when compared to a direct out-of-plane upper bound approach conducted with finite elements.

According to the layered approach, the RVEs considered for the determination of the in-plane strength domains are here re-analysed, subdividing them along the thickness into several layers (for practical purposes, it has been shown that 12 subdivisions represent a good compromise between numerical efficiency and reliability of the results). For each layer, the out-of-plane stress components $\sigma_{\mathrm{i} 3}(\mathrm{i}=1,2,3)$ of the micro-stress tensor $\sigma$ are set to zero, meaning that only the in-plane components $\sigma_{\mathrm{ij}}(\mathrm{i}, \mathrm{j}=1,2)$ are considered active and constant along the $\Delta_{i l}$ thickness. This allows handling the homogenized out-of-plane strength domain in each layer as it was in plane stress-

condition. The out-of-plane strength domain $S^{\text {hom }}$ may be therefore estimated by means of the following optimization problem: 


$$
\left\{\begin{array}{c}
\max \{\lambda\} \\
\mathbf{N}=\int_{k, i_{L}}^{k} \tilde{\boldsymbol{\sigma}}^{\left(i_{L}\right)} d V \\
\mathbf{M}=\int_{k, i_{L}}^{k} y_{3} \tilde{\boldsymbol{\sigma}}^{\left(i_{L}\right)} d V \\
\mathbf{s u c h} \text { that }\left\{\begin{array}{cc}
M_{11} & M_{12} \\
M_{12} & M_{22}
\end{array}\right]=\lambda\left[\begin{array}{cr}
\cos (\psi) \cos (\vartheta) & \sin (\vartheta) \\
\sin (\vartheta) & \sin (\psi) \cos (\vartheta)
\end{array}\right] \\
\psi=[0 ; 2 \pi] \\
\theta=[0 ; \pi / 2] \\
\tilde{\boldsymbol{\sigma}}^{\left(i_{L}\right)} \in S^{i_{L}} \\
i_{L}=1, \ldots, \text { number of layers }
\end{array}\right.
$$

where:

- $\lambda$ is the load multiplier, representing the ultimate moment in the $M_{11}-M_{22}-M_{12}$ (horizontal bending-vertical bending-torsion) along the direction $\mathbf{n}_{\Sigma}$ identified by angles $\psi$ and $\vartheta$;

- $\tilde{\boldsymbol{\sigma}}^{\left(i_{L}\right)}$ denotes the homogenized membrane stress tensor (i.e. $\tilde{\boldsymbol{\sigma}}^{\left(i_{L}\right)}=\left[\begin{array}{lll}\tilde{\sigma}_{11}^{\left(i_{L}\right)} & \tilde{\sigma}_{12}^{\left(i_{L}\right)} ; \tilde{\sigma}_{12}^{\left(i_{L}\right)} & \tilde{\sigma}_{22}^{\left(i_{L}\right)}\end{array}\right]$ ) of layer $i_{L}$

- $\psi$ and $\vartheta$ are spherical coordinates in $M_{11}-M_{22}-M_{12}$, given by $\tan (\vartheta)=\frac{M_{12}}{\sqrt{\left(M_{11}^{2}+M_{22}^{2}\right)}}$, $\tan (\psi)=\frac{M_{22}}{M_{11}}$

- $S^{i_{L}}$ denotes the (non-linear) homogenized strength domain of the $i_{L}^{\text {th }}$ layer.

Membrane actions are kept for the sake of simplicity constant and independent from load multiplier. In particular, we assume that $N_{11}=N_{12}=0$ and that $N_{22}=-\gamma(H-z) t$ where $\gamma$ is masonry specific weight, $H$ and $t$ are wall height and thickness and $z$ is the vertical position of the RVE considered. Such hypothesis on membrane loads seems reasonable for practical purposes and approximates well real in-plane internal actions for laboratory tests and isolated walls or walls with a few perpendicular interconnections, as the case here analyzed. Finally, when dealing with the analysis at collapse of the Alcaçova wall reported in what follows, it is worth mentioning that inplane vertical pre-compression affects considerably $M_{11}, M_{22}, M_{12}$ strength domains. This assumption proved to be technically acceptable in the majority of the real cases. 
In the out-of-plane numerical simulations, the thickness of the RVEs is assumed equal to $0.40 \mathrm{~m}$ and three increasing compressive vertical loads are analysed, corresponding to three different levels with respect to the top edge of the Alcaçova wall: $N_{22}=0$ (top), $N_{22}=$ self-weight/2 (mid-height), $N_{22}=$ self-weight (bottom). Out-of-plane failure surfaces are presented for the sake of clearness as sections on $M_{11}-M_{22}$ and $M_{11}-M_{12}$ planes.

In Figure 21 and Figure 23, $M_{11}-M_{22}$ (sub-figures -a) and $M_{11}-M_{12}$ (sub-figures -b) out-ofplane homogenized failure surface section for the $4 x 4 a$ RVE at increasing vertical compressive loads are depicted, for mortar with poor and good mechanical properties respectively.

In Figure 22, for the sake of completeness, two 3D representations of the out-of-plane failure surface ( $M_{11}-M_{22}-M_{12}$ space) for the 4 x4a RVE, assuming respectively a zero (-a) and maximum (-b) pre-compression are depicted. The maximum pre-compression occurs in correspondence of the wall foot, whereas a zero pre-compression is present at the top edge. Analogously to the in-plane case, the orthotropic behaviour of the masonry material considered, again linked to blocks staggering, is worth noting. Furthermore, pre-compression tends to slightly distort the orthotropic failure surface when $M_{11}$ and $M_{22}$ have opposite signs.

As expected, the vertical pre-compression increases not only the vertical bending moment $\left(M_{22}\right)$ but also the horizontal one, as a result of the interlocking of the blocks and the frictional behaviour of the bed joint, which exhibits an increased torsional resistance when pre-compressed. In some cases, with strong mortar (3x3b, 3x3c, 4x4c, 5x5b), due to the insufficient staggering of the stones in the RVE, $M_{11}$ does not increase sensibly and a straight vertical crack is obtained, meaning that a blocks failure is also experienced. In general, authors experienced that very similar results are obtained performing calculations ( 4 ) on all the different RVEs considered in the present study, see Figure 12.

In Figure 24 (-a: weak mortar, b: strong mortar), a comparison between the mean values of out-ofplane strengths obtained averaging results of different RVEs of the same size is depicted, assuming 
a compressive load $N_{22}=132.8 \mathrm{kN} / \mathrm{m}$, corresponding to the situation at the base of the wall. As can be observed, the vertical bending moment $\left(M_{22}\right)$ exhibits very similar values for the different cell sizes (and the same occurs for torsion $M_{12}$ ). Some non-negligible perturbation is observed for horizontal bending moment $\left(M_{11}\right)$, being more sensitive to the compressive loads and the actual staggering of the blocks. Still, the scatter is fully acceptable for engineering purposes.

A good benchmark to evaluate the reliability of results provided by the numerical models presented in this study, is obtained comparing the ultimate vertical bending moments $M_{22}$ provided by the present analysis with those obtained by means of the following simple equation, see [14], which is deduced by simple equilibrium considerations assuming a flexural failure occurring on a single bed joint:

$$
\begin{aligned}
& M_{22}=\frac{x(t-x)}{2}\left(f_{c}+f_{t}\right) \\
& x=\frac{f_{c} t+N_{22}}{f_{c}+f_{t}}
\end{aligned}
$$

A comparison between homogenization predictions and equation ( 5 ) results is reported in Figure 25 for both weak (-a) and strong (-b) mortar, as a function of the vertical pre-compression $N_{22}$. The correspondence is almost perfect for all the cases considered, meaning that reliable approximations might be achieved using the numerical model presented in this study.

\section{Structural analyses}

For the sake of conciseness, the structural study is restricted to the part of the Alcaçova wall located above a much thicker wall (see Figure 2) connected to secondary small buildings and rooms. The analysis of the whole wall would require full 3D computations, which are outside the scope of the present paper. 
The portion of the wall under consideration may be reasonably assumed as supported on three edges, one corresponding to the lower edge, the remaining two corresponding to vertical boundaries. The dimensions of the wall are $14.25 \mathrm{~m}$ in length and $6.85 \mathrm{~m}$ in height. The thickness of the external leaves is $0.40 \mathrm{~m}$. The wall has seven openings, labelled from O-1 to O-7 for the sake of clearness in Figure 2-c. The openings represent approximately $20 \%$ of the area of the entire wall. Above each opening a lintel is present.

In order to carry out the analysis of Alcaçova wall, two numerical models are utilized and the results obtained critically compared: the first is a heterogeneous model, where the actual disposition of the blocks is considered in detail, whereas the second is a homogenized model where strength domain obtained in the previous section are utilized. Figure 26 shows the discretization adopted for both models and the number of elements required for the analyses.

Limit analyses are performed using the out-of-plane model firstly proposed in [47], where plastic dissipation is allowed only at the interfaces between adjoining elements. Such approach is sufficiently reliable in the case under consideration because blocks may be reasonably regarded as infinitely resistant, whereas cracks are expected to zigzag directly inside thin joints. Non negligible differences are expected if comparing the results obtained with those for regular patterns, because the actual disposition of the blocks may play a major role, especially in horizontal bending. For instance, when blocks staggering decreases between two contiguous rows for some irregularities of block dimensions, the overall horizontal bending strength in that region decreases considerably, because horizontal joint contribution tends to vanish.

Results obtained comprise limit loads and the possible collapse mechanisms, whereas no information on displacements is provided. External seismic load depending on the load multiplier is applied to the model as out-of-plane pressure on single elements following a first mode distribution. Collapse mechanism obtained by means of both the heterogeneous and the homogenized model assuming joints with weak mortar are depicted in Figure 28. The actual thickness of the plate 
elements is visualized for the sake of clearness, as well as all the cracks forming the failure mechanism are labelled.

Cracks a4, a5, a13, a14, a17 belong to two types of vertical cracks located in the middle third of the wall. These cracks are caused by the vertical bending moment. Crack a4 is a vertical line and forms for the inexistent staggering of the blocks in eleven masonry courses in this area. Crack a13 propagates following head and bed joints, whereas Cracks a8 and a18 follow the horizontal alignment of the blocks. Cracks a1, a2, a3, a6, a7, a9, a10, a11, a12, a15, a16 appear on corners and surrounding different portions of the openings, and are mainly diagonal, propagating for a combined effect of horizontal and vertical bending moment. This combination of effects is caused by the edge constrains applied to the wall, representing the connections of the Alcaçova wall with orthogonal walls and with the base horizontal base. It is important to remark that these types of cracks develop on areas close to the lateral edges. Finally, it can be stated that globally the failure mechanism is similar to a local overturning, as usually occurs for historical masonry buildings.

When dealing with the homogeneous model, it should be taken in to account that cracks can only follow the mesh lines. As can be seen from Figure 28-b, where the failure mechanism obtained by means of the homogenized model is represented, such model appears in term of cracks, in satisfactory agreement with the heterogeneous one. In particular, crack b1 is similar to a1, b2 to a2, b3 to a3. b4 appears slightly different and propagates on the right side, but maintaining the same pattern (vertical crack). b5 is again similar to a5, a6 to b6, b7 to a9, b8 to a11, b14 to a19, b15 to a14, b16 to b15, b17 to a16, b18 to a18 and b19 to a17. Cracks b10, b11, b12 and b20 exhibit some discrepancies. However, from a global point of view, the agreement seems quite satisfactory, both models providing comparable failure mechanisms.

Figure 29 shows the same comparison on collapse mechanisms for a strong mortar condition. Again, the collapse active mechanisms are characterized in both cases by a long vertical crack on the right, developing on the same eleven head joints. Furthermore, horizontal cracks are still present 
in the corners of the openings, as the compressive vertical load provided by self-weight is not sufficient to preclude cracks promoted by the horizontal bending.

Diagonal cracks for combined vertical and horizontal bending are still present near the edges of the wall and also in the corners of openings. As can be seen, the cracks patterns provided by the heterogeneous and homogenized model appear again in very good agreement. In particular, the following matches hold:c1 is comparable with $d 1$, c2 with d2, c3 with d3, c4 with d6, c21 with d7, c6 with d8, c20 with d18, c8 with d10, c9 with d19, c10 with d20, c11 with d11, c12 with d12, c18 with d13, c16 with d14, c15 with d15, c14 with d21, c17 with d16.

A comparison between the failure loads obtained with both models is summarized in Table 5 , for both strong and weak mortar hypotheses. The accuracy of the homogenized model, defined as the ratio between the homogenized and heterogeneous failure loads, is also represented. As can be noted, homogenized limit analysis results are in good agreement with the full heterogeneous computations, confirming the reliability of the approach proposed. Limit load is represented as a fraction of the wall self-weight, thus giving a direct indication of the horizontal acceleration that the structure may withstand under seismic excitation, as a fraction of the gravity acceleration. Obviously, for weak mortar (the most realistic assumption) the vulnerability of the wall under horizontal loads results quite high.

In order to further assess the results obtained at a structural level with the model proposed, a sensitivity analysis upon mesh refinement is conducted, with the three different meshes reported in Figure 27. A fast convergence of results is obtained, represented in terms of accuracy of the homogenized model when compared to the heterogeneous one. As can be seen, sensitivity to mesh refinement is rather low. Indeed, no plastic deformation is allowed in the model, which essentially is a FE implementation of the yield line theory. A slight dependence of collapse loads upon both interfaces orientation and disposition is therefore expected. Mesh adaptation schemes based on sequential linear programming, after [48], are under study by the authors. 
It is worth emphasizing that the crack patterns provided by Finite Elements with strong mortar keep a certain similarity to the ones obtained in the case of weak mortar. The main reason is that the failure mode is heavily controlled by the geometrical restraints. The purpose of using a strong mortar (i.e. a lime grout based injection during a possible rehabilitation intervention) is indeed not to change the structural behaviour of the wall, but to improve the ultimate load.

Finally, it has to be underlined that the limit load associated to the heterogeneous model is equal to $34.4 \%$ of the self-weight, whereas the limit load of the homogeneous model is equal to $31.6 \%$ of the self-weight. The results seem in reasonable agreement (less than 10\% difference, see Table 5 ), with closer match between the original and the strengthened wall. It is also interesting to observe that the introduction of strong mortar significantly increases the limit load of the Alcaçova wall (almost four times).

\section{Conclusions}

The behaviour at collapse of one perimeter wall belonging to the Guimarães castle in Portugal has been investigated by means of a rigid-plastic homogenization procedure, accounting for the actual disposition of the blocks constituting the wall and the texture irregularity given by the variability of dimensions in the blocks.

To determine such geometric variability, a detailed geometric survey has been conducted by means of the laser scanning technique, allowing for a precise characterization of dimensions and disposition of the blocks.

Laser scanning technique was proved to be useful for the accurate and extensive geometric survey of large masonry structures, as the example of Guimaraes castle. Additionally, a novel procedure that avoids the tedious and costly data processing was developed, allowing automatic geometric extraction of masonry blocks. This achievement facilitates integrating such fast, massive and accurate geometric tool in structural engineering applications because an expert in geomatics is not required to manage the massive data. 
After a simplification of the geometry and assuming mortar joints reduced to interfaces, homogenized masonry in- and out-of-plane strength domains have been evaluated on a number of different Representing Elements of Volume (RVEs) having different sizes and sampled on the walls of the castle. Strength domains have been obtained using a Finite Element (FE) limit analysis approach with a heterogeneous discretization of the RVEs.

By means of such strength domains, a homogenized limit analysis has been carried out on the wall and results have been compared with those provided by a standard heterogeneous discretization of the domain. The comparison, in presence of both good and weak mortar joints, have proved a satisfactory reliability of the homogenization proposed performed on different RVEs with blocks having variable dimension.

\section{References}

[1] Lotfi HR, Shing PB (1994). Interface model applied to fracture of masonry structures. Journal of Structural Engineering ASCE 120 (1): 63-80.

[2] Lourenço PB, Rots J (1997). A multi-surface interface model for the analysis of masonry structures. Journal of Engineering Mechanics ASCE 123 (7): 660-668.

[3] Milani G (2008). 3D upper bound limit analysis of multi-leaf masonry walls. International Journal of Mechanical Sciences 50: 817-836.

[4] Milani G, Zuccarello FA, Olivito RS, Tralli A (2007). Heterogeneous upper-bound finite element limit analysis of masonry walls out-of-plane loaded. Computational Mechanics 40 (6): 911-931.

[5] Oliveira DV, Lourenço PB (2004). Implementation and validation of a constitutive model for the cyclic behaviour of interface elements. Computers \& Structures 82(17-19): 1451-1461.

[6] Milani G, Lourenço PB (2010). Monte Carlo homogenized limit analysis model for randomly assembled blocks in-plane loaded. Computational Mechanics 46(6): 827-849

[7] Milani G., Benasciutti D. (2010). Homogenized limit analysis of masonry structures with random input properties: polynomial response surface approximation and Monte Carlo simulations. Structural Engineering and Mechanics 34(4): 417-447.

[8] Milani G., Lourenço P.B. (2010). A simplified homogenized limit analysis model for randomly assembled blocks out-of-plane loaded. Computers \& Structures 88: 690-717.

[9] Milani G. (2011). Simple lower bound limit analysis homogenization model for in- and outof-plane loaded masonry walls. Construction \& Building Materials, 25: 4426-4443.

[10] Pegon P, Anthoine A (1997). Numerical strategies for solving continuum damage problems with softening: application to the homogenization of masonry. Computers and Structures 64(4): 623-642. 
[11] Luciano R, Sacco E (1997). Homogenization technique and damage model for old masonry material. International Journal of Solids and Structures 34(4): 3191-208.

[12] Pietruszczak S, Ushaksaraei R (2003). Description of inelastic behaviour of structural masonry. International Journal of Solids and Structures 40: 4003-4019.

[13] Massart T, Peerlings RHJ, Geers MGD (2004). Mesoscopic modeling of failure and damageinduced anisotropy in brick masonry. European Journal of Mechanics A/Solids 23: 719-735.

[14] Milani G (2011). Simple homogenization model for the non-linear analysis of in-plane loaded masonry walls. Computers \& Structures 89: 1586-1601.

[15] Lourenço PB, Milani G, Tralli A, Zucchini A (2007). Analysis of masonry structures: Review of and recent trends of homogenisation techniques. Canadian Journal of Civil Engineering 34(11): 1443-1457.

[16] Formica G, Sansalone V, Casciaro R (2002). A mixed solution strategy for the nonlinear analysis of brick masonry walls. Computer Methods in Applied Mechanics and Engineering 191(51-52): 5847-5876.

[17] Gambarotta L, Lagomarsino S (1997). Damage models for the seismic response of brick masonry shear walls. Part I: the mortar joint model and its applications. Earthquake Engineering and Structural Dynamics 26: 423-439.

[18] Lourenço PB, Rots JG, Blaauwendraad J (1998). Continuum model for masonry: Parameter estimation and validation. Journal of Structural Engineering ASCE 124(6): 642-652.

[19] Brasile S, Casciaro R, Formica G (2010). Finite Element formulation for nonlinear analysis of masonry walls. Computers \& Structures 88(3-4): 135-143

[20] Cluni F, Gusella V (2004). Homogenization of non-periodic masonry structures. International Journal of Solids and Structures 41: 1911-1923.

[21] Falsone G, Lombardo M (2007). Stochastic representation of the mechanical properties of irregular masonry structures. International Journal of Solids and Structures 44: 8600-8612.

[22] Cecchi A, Sab K (2009). Discrete and continuous models for in plane loaded random elastic brickwork. European Journal of Mechanics A-Solids 28: 610-625.

[23] Gusella V, Cluni F (2006). Random field and homogenization for masonry with nonperiodic microstructure. Journal of Mechanics of Materials and Structures 1(2): 357-386.

[24] Cecchi A, Sab K (2009). A homogenized Love-Kirchhoff model for out-of-plane loaded Random 2D lattices. Application to Brickwork Panels. International Journal of Solids and Structures 46(14-15): 2907-2919.

[25] Lombardo M, Zeman J, Šejnoha M, Falsone G (2009). Stochastic modeling of chaotic masonry via mesostructural characterization. International Journal for Multiscale Computational Engineering 7(2): 171-185.

[26] Zeman J, M. Šejnoha M (2007). From random microstructures to representative volume elements. Modelling Simulation Mater. Sci. Eng. 15(4): 325-335.

[27] Šejnoha J, Šejnoha M, Zeman J, Sýkora J, Vorel J (2008). A mesoscopic study on historic masonry. Structural Engineering \& Mechanics 30 (1): 99-117.

[28] Cavalagli N, Cluni F, Gusella V (2011). Strength domain of non-periodic masonry by homogenization in generalized plane state. European Journal of Mechanics A/Solids, 30: 113126 
[29] Zeman J, Šejnoha M (2001). Numerical evaluation of effective properties of graphite fiber tow impregnated by polymer matrix, Journal of the Mechanics and Physics of Solids 49 (1): 69-90.

[30] Suquet P (1983). Analyse limite et homogeneisation. Comptes Rendus de l'Academie des Sciences - Series IIB - Mechanics 296: 1355-1358.

[31] Milani G, Lourenço PB, Tralli A (2006). Homogenised limit analysis of masonry walls - Part I: failure surfaces. Computers and Structures 84: 166-180.

[32] de Buhan P, de Felice G (1997). A homogenization approach to the ultimate strength of brick masonry. Journal of the Mechanics and Physics of Solids, 45 (7): 1085-1104.

[33] Milani G, Milani E, Tralli A (2009). Upper Bound limit analysis model for FRP-reinforced masonry curved structures. Part I: unreinforced masonry failure surfaces. Computers \& Structures 2009; 87 (23-24): 1516-1533.

[34] Heyman J. (1969). The safety of masonry arches. International Journal of Mechanical Sciences 43: 209-224.

[35] Huet C (1990). Application of variational concepts to size effects in elastic heterogeneous bodies. Journal of the Mechanics and Physics of Solids 38 (6): 813-841.

[36] Sab K (1992). On the homogenization and the simulation of random materials. European Journal of Mechanics, A/Solids 11 (5): 585-607.

[37] Gitman IM, Askes H, Sluys LJ (2007) Representative volume: Existence and size determination. Engineering Fracture Mechanics 74(16): 2518-2534.

[38] Sloan SW, Kleeman PW (1995). Upper bound limit analysis using discontinuous velocity fields. Computer Methods in Applied Mechanics and Engineering 127 (1-4): 293-314.

[39] Milani E, Milani G, Tralli A (2008). Limit analysis of masonry vaults by means of curved shell finite elements and homogenization. International Journal of Solids and Structures 45: 5258-5288.

[40] Milani G, Beyer K., Dazio A. (2009). Upper bound limit analysis of meso-mechanical spandrel models for the pushover analysis of 2D masonry frames. Engineering Structures, 31(11), pp. 2696-2710.

[41] González-Jorge H, Riveiro B, Armesto J, Arias P (2012). Procedure to evaluate the accuracy of laser-scanning systems using a linear precision electro-mechanical actuator.IET Science, Measurement and Technology 6(1): 6-12.

[42] Höfle B, Pfeifer N (2007). Correction of laser scanning intensity data: Data and model-driven approaches. ISPRS Journal of Photogrammetry \& Remote Sensing 62: 415-433.

[43] Awcock GJ and R. Thomas R (1996). Applied Image processing. Ed. McGraw-Hill, 300 pp.

[44] Vasconcelos G, Lourenço PB, Alves CAS and Pamplona, J 2008. Experimental characterization of the tensile behaviour of granites. International Journal of Rock Mechanics and Mining Sciences 45(2): 268-277.

[45] Dhanasekar M, Page A, Kleeman P (1985). The failure of brick masonry under biaxial stresses, Proc. Instn Civ. Engrs: 295-313.

[46] Milani G, Lourenco PB, Tralli A (2006). Homogenization approach for the limit analysis of out-of-plane loaded masonry walls. Journal of Structural Engineering ASCE, 132(10): 16501663. 
[47] Cecchi A, Milani G, Tralli A (2007). A Reissner-Mindlin limit analysis model for out-ofplane loaded running bond masonry walls. International Journal of Solids and Structures, 44(5): 1438-1460.

[48] Milani G, Lourenço PB (2009). A discontinuous quasi-upper bound limit analysis approach with sequential linear programming mesh adaptation. International Journal of Mechanical Sciences 51(1): 89-104.

\section{APPENDIX A: FE limit analysis model}

To provide collapse loads for the RVEs reported in Figure 12 and Figure 13, a FE limit analysis code is adopted, which utilizes a triangular discretisation with discontinuities of the velocity field along the edges of adjacent triangles. At each node $j$, a horizontal velocity $u_{x}^{j}$ and a vertical velocity $u_{y}^{j}$ are introduced. The resulting velocity field within a triangular element is linear, whereas the strain rate field is constant. Across the interfaces a linear velocity jump is assumed and therefore for each interface four unknowns are introduced ( $\Delta \mathbf{u}=\left[\Delta v^{1} \Delta u^{1} \Delta v^{2} \Delta u^{2}\right]^{T}$ ) representing the normal $\left(\Delta v^{j}\right)$ and tangential $\left(\Delta u^{j}\right)$ velocity jumps with respect to the discontinuity direction evaluated at the nodes $j=1$ and $j=2$ of the interface. For any pair of nodes on the interface between two adjacent triangles $(m)-(n)$, the tangential and normal velocity jumps can be written in terms of the Cartesian nodal velocities of elements $(m)-(n)$, so that four linear equations in the form $\mathbf{A}_{11}^{e q} \mathbf{u}^{E m}+\mathbf{A}_{12}^{e q} \mathbf{u}^{E n}+\mathbf{A}_{13}^{e q} \Delta \mathbf{u}=\mathbf{0}$ can be written for each interface, where $\Delta \mathbf{u}$ has been already defined, $\mathbf{u}^{E m}$ and $\mathbf{u}^{E n}$ are the $6 x 1$ vectors that collect the velocities of the elements $(m)$ and $(n)$ respectively, $\mathbf{A}_{11}^{e q}$ and $\mathbf{A}_{12}^{e q}$ are $4 \times 6$ matrices depending only on nodal coordinates of element $(\mathrm{m})$ and $(n)$, respectively, and $\mathbf{A}_{13}^{e q}$ is a $4 \times 4$ matrix depending only on coordinates of nodes 1 and 2.

For continuum, three equality constraints representing the plastic flow in continuum (obeying an associated flow rule) are introduced for each element in the form: 
$\dot{\boldsymbol{\varepsilon}}_{p l}^{E}=\left[\frac{\partial u_{x}}{\partial x} \frac{\partial u_{y}}{\partial y} \frac{\partial u_{y}}{\partial x}+\frac{\partial u_{x}}{\partial y}\right]=\dot{\lambda}^{E} \frac{\partial S^{C}}{\partial \boldsymbol{\Sigma}^{C}}$

where $\dot{\boldsymbol{\varepsilon}}_{p l}^{E}$ is the plastic strain rate vector of element $E, \dot{\lambda}^{E} \geq 0$ is the plastic multiplier, $S^{C}$ indicates a generic (non) linear failure surface for continuum and $\boldsymbol{\Sigma}^{C}=\left[\begin{array}{lll}\sigma_{x} & \sigma_{y} & \tau\end{array}\right]^{T}$ is the plane-stress vector in continuum ( $\sigma_{x}$ : normal $x$-axis stress, $\sigma_{y}$ : normal $y$-axis stress, $\tau$ : tangential stress).

Assuming that a linearization with $m$ planes of the failure surface in the form $S^{C} \equiv \mathbf{A}^{\text {in }} \boldsymbol{\Sigma}^{C} \leq \mathbf{b}^{\text {in }}$ is available, where $\mathrm{A}^{\text {in }}$ is a $m x 3$ matrix (each row corresponds to coefficients of one linearization plane) and $\mathbf{b}^{\text {in }}$ is the $m x 1$ vector of linearization planes right hand sides, the three linear equality constraints per element can be written in the form $\mathbf{A}_{21}^{e q} \mathbf{u}^{E}+\left(\mathbf{A}^{i n}\right)^{T} \dot{\lambda}^{E}=\mathbf{0}$, where $\mathbf{u}^{E}$ is the vector of element velocities, $\dot{\lambda}^{E}$ is the $m x 1$ vector of plastic multiplier rates of the element (one for each plane of the linearized failure surface), and $\mathbf{A}_{21}^{\text {eq }}$ is a $3 \times 6$ matrix depending only on nodal coordinates of the element.

Analogously to the continuum case, one can assume that a linearized failure surface $S^{I} \equiv \mathbf{A}_{I}^{i n} \boldsymbol{\Sigma} \leq \mathbf{b}_{I}^{i n}$ is available for the interfaces (which typically coincide with mortar joints reduced to interfaces or internal brick interfaces). Here $\boldsymbol{\Sigma}=\left[\begin{array}{ll}\sigma & \tau\end{array}\right]^{T}$ collects the normal stress $\sigma$ on the interface and the shear action $\tau$ in the interface direction, while the matrices $\mathbf{A}_{I}^{\text {in }}$ and $\mathbf{b}_{I}^{\text {in }}$ have the same meaning as in the continuous case.

For the particular problem at hand, further equalities constraints in the general matrix form:

$$
\mathbf{A}_{j r}^{e q} \mathbf{u}^{E p}+\mathbf{A}_{j p}^{e q} \mathbf{u}^{E r}=\mathbf{0}
$$

must be applied in the numerical model for piers and spandrel in order to comply with particular boundary conditions specified. 
After some elementary assemblage operations, a simple linear programming problem is obtained where the objective function is the total internal power:

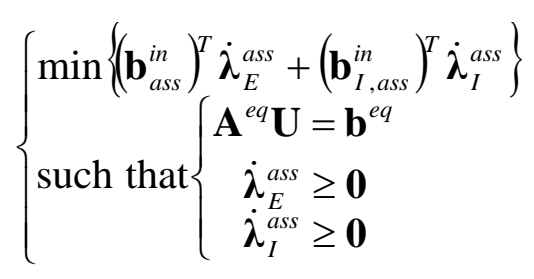

where:

- $\mathbf{b}_{\text {ass }}^{\text {in }}$ and $\mathbf{b}_{I, \text { ass }}^{\text {in }}$ are the assembled right-hand sides of the inequalities, which determine the linearised failure surface of the material of the continuum and of the interfaces, respectively;

- $\mathbf{U}=\left[\begin{array}{llll}\mathbf{u} & \dot{\lambda}_{E}^{\text {ass }} & \Delta \mathbf{u}^{\text {ass }} & \dot{\lambda}_{I}^{\text {ass }}\end{array}\right]$ is the vector of global variables, which collects the vector of assembled nodal velocities ( $\mathbf{u}$ ), the vector of assembled element plastic multiplier rates $\left(\dot{\lambda_{E}}\right.$ ass , the vector of assembled velocity jumps on interfaces ( $\left.\Delta \mathbf{u}^{\text {ass }}\right)$, and the vector of assembled interface plastic multiplier rates $\left(\dot{\boldsymbol{\lambda}}_{I}^{\text {ass }}\right)$;

- $\mathrm{A}^{\text {eq }}$ is the overall constraints matrix and collects velocity boundary conditions, relations between velocity jumps on interfaces and elements velocities, constraints for plastic flow in velocity discontinuities, constraints for plastic flow in continuum and ( 7 ) equalities. 


\section{Figures}

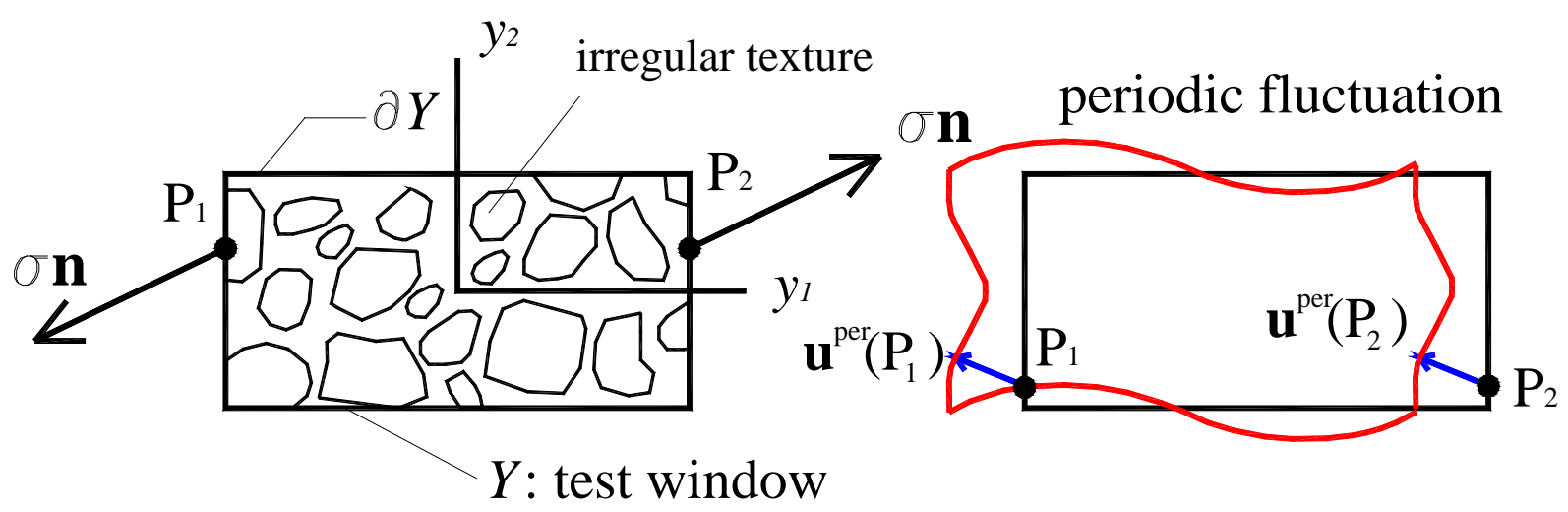

Figure 1: Generic test-window on a irregular texture, anti-periodicity of the micro-stress field and periodicity of $\mathbf{u}^{\mathrm{per}}$. 


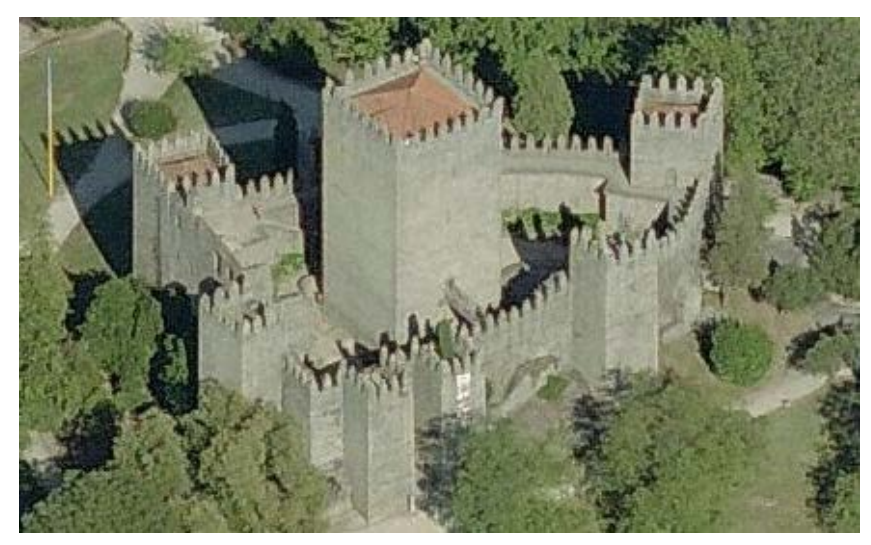

$-\mathrm{a}$

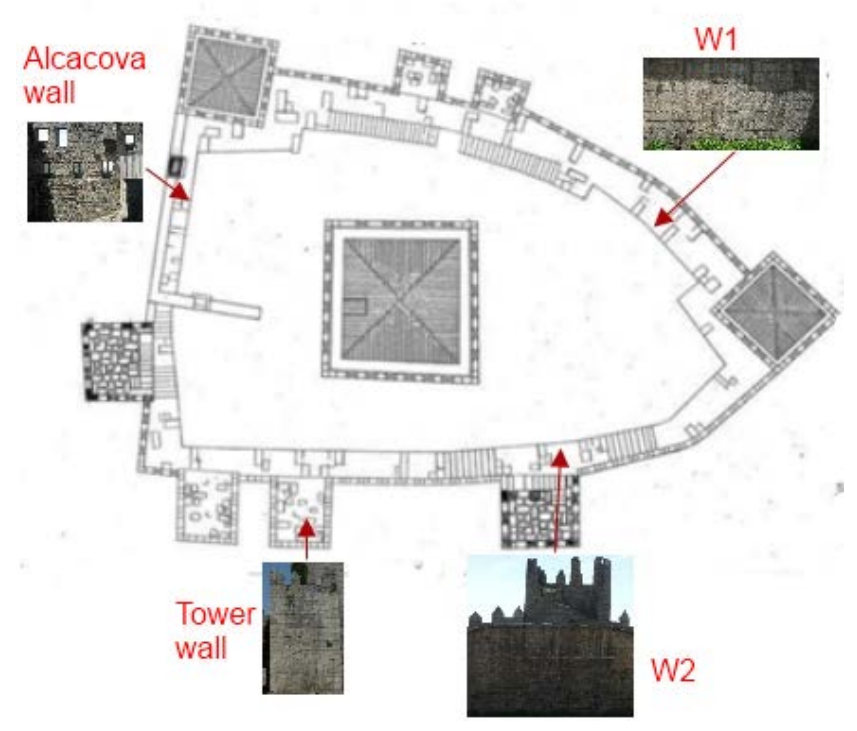

$-b$

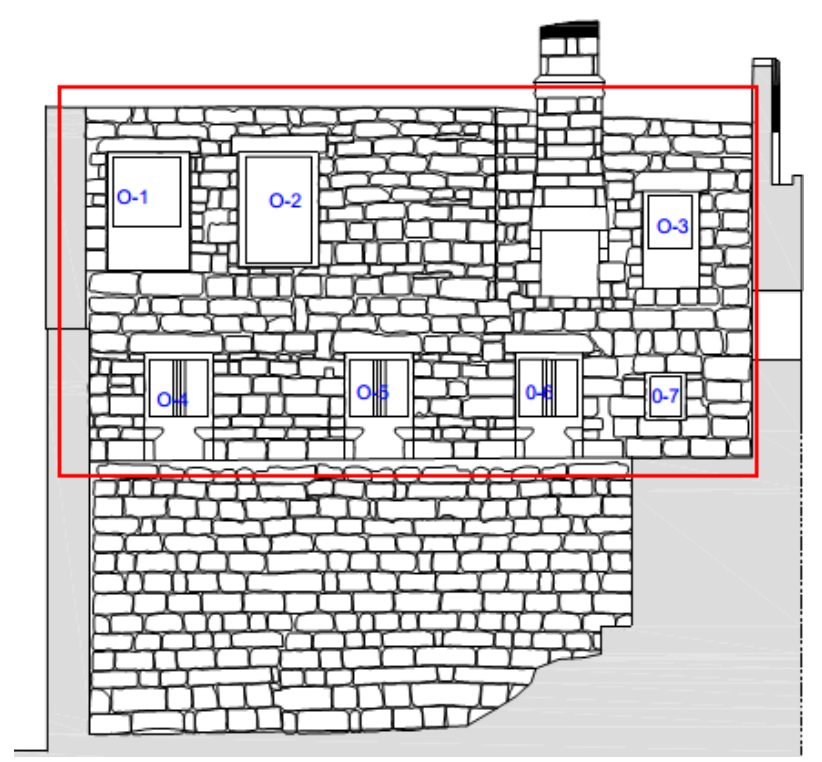

$-\mathrm{C}$

Figure 2. Guimarães castle. -a: Panoramic View. -b: Plan view. -c:

Alcaçova wall: sector enclosed in the red box is considered in the numerical models. 


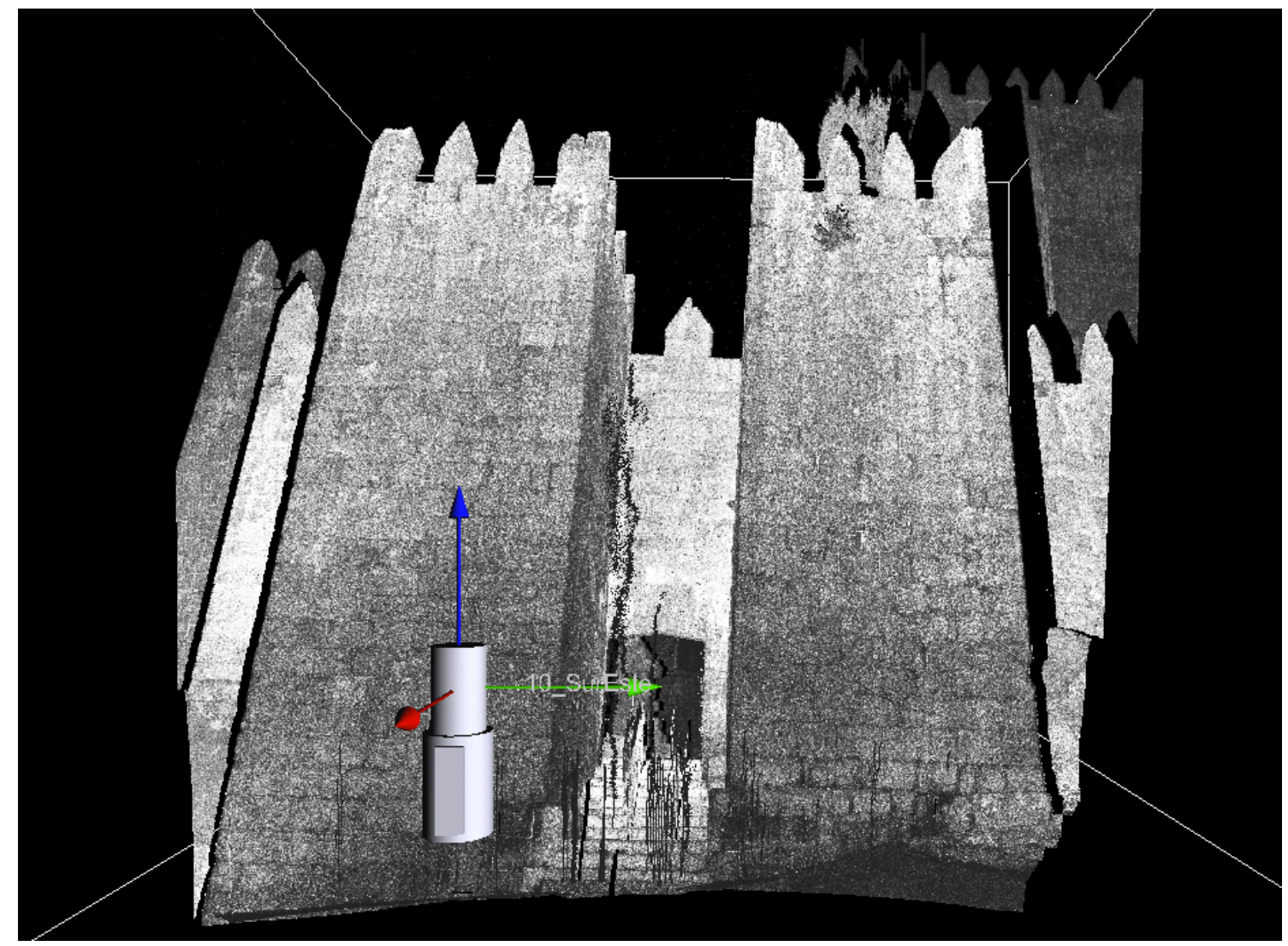

Figure 3. Raw point cloud of two of the tower of the castle coloured with the attribute of intensity for each points. 


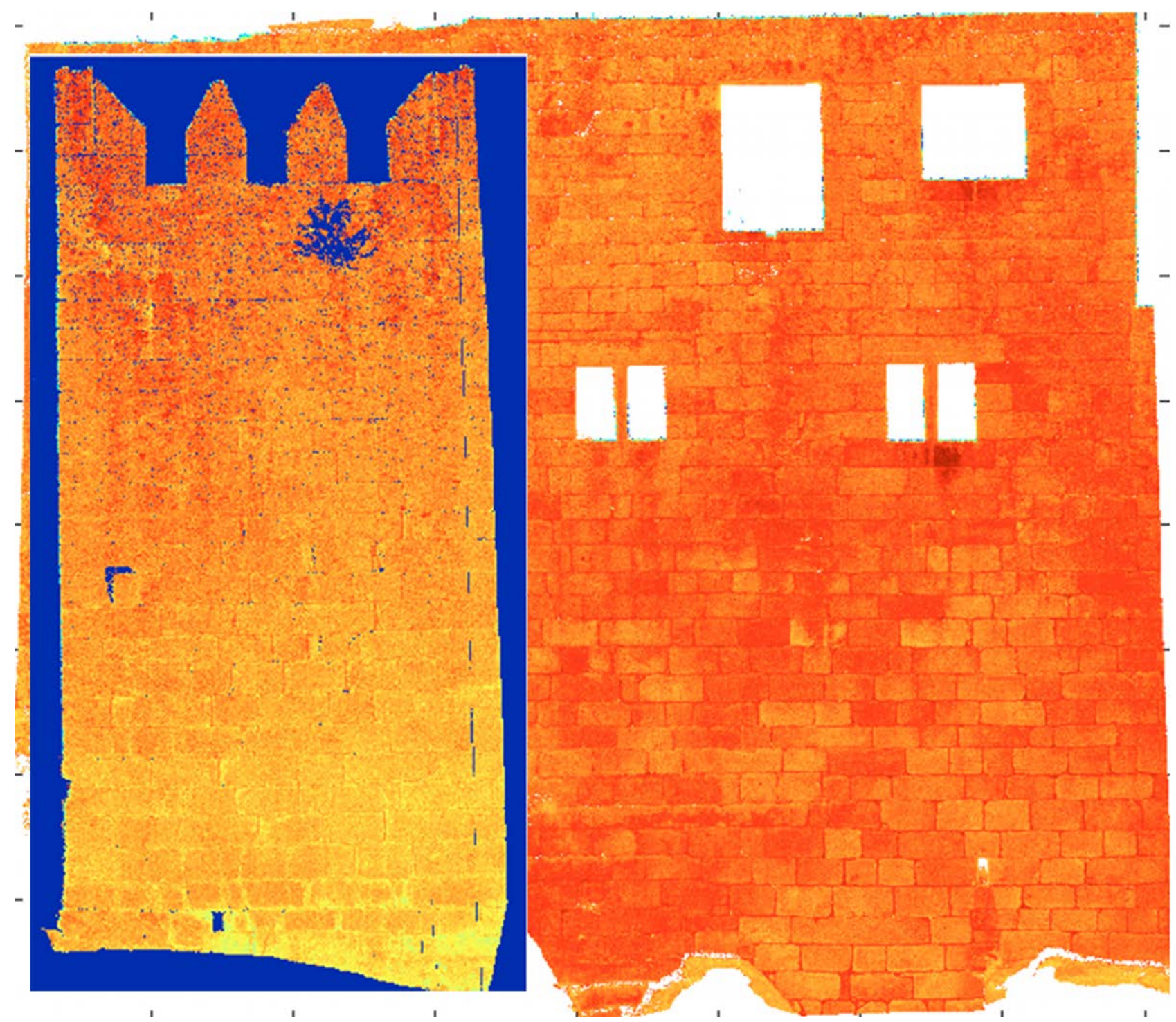

Figure 4. Intensity image of Alcaçova wall (background) and one tower (front). 


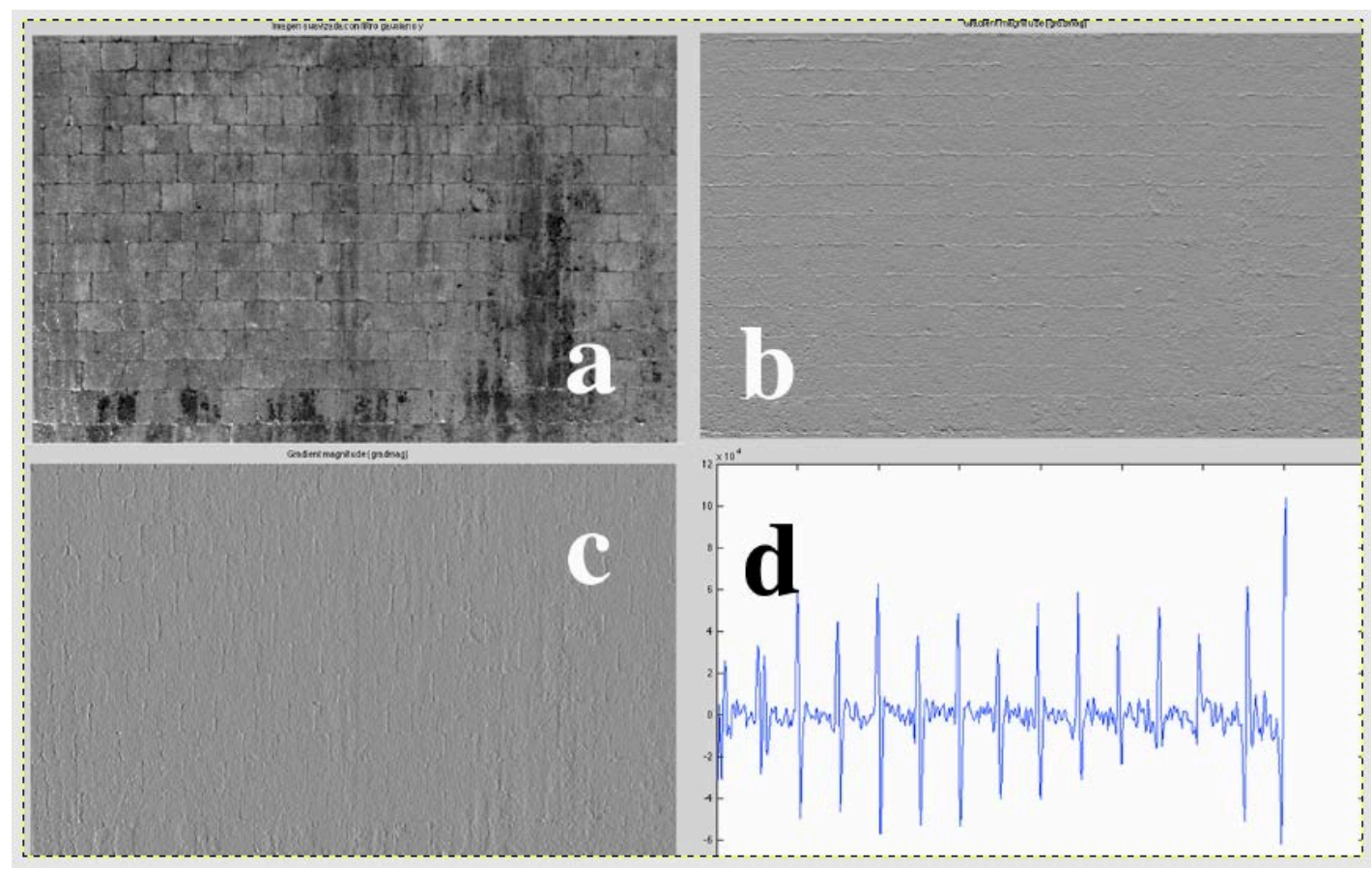

Figure 5. Edge detection process for a window sample in the walls: a) grey scaled intensity image; b) first derivatives for horizontal direction; c) first derivatives for vertical direction; d) peaks showing joints between masonry courses. BELEN: size of a b c d to reduce, increase font on subfigures and in english 


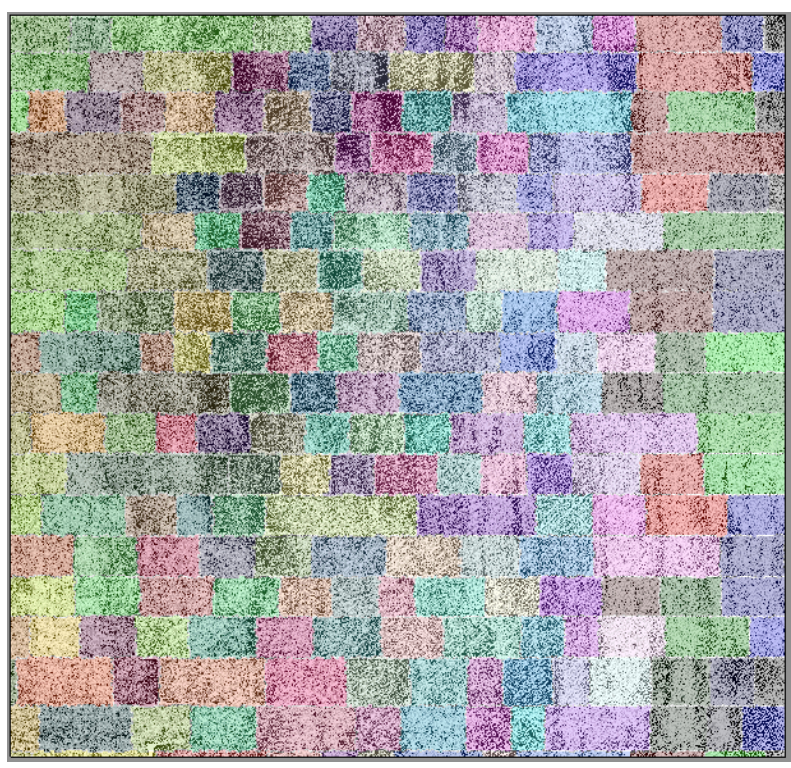

$-\mathrm{a}$

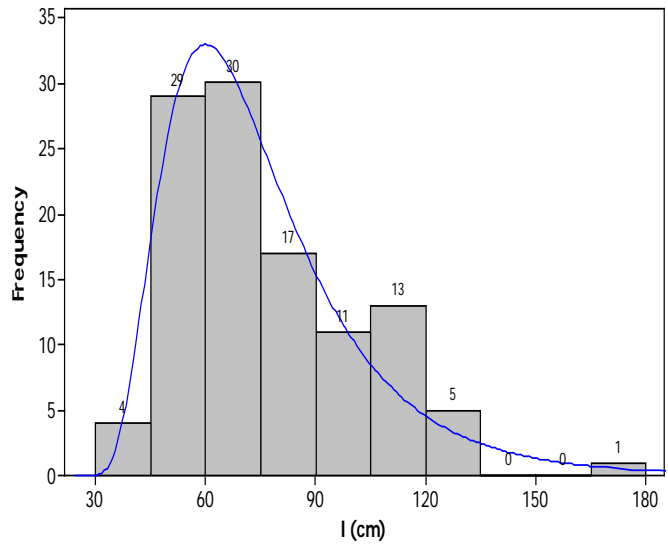

-b

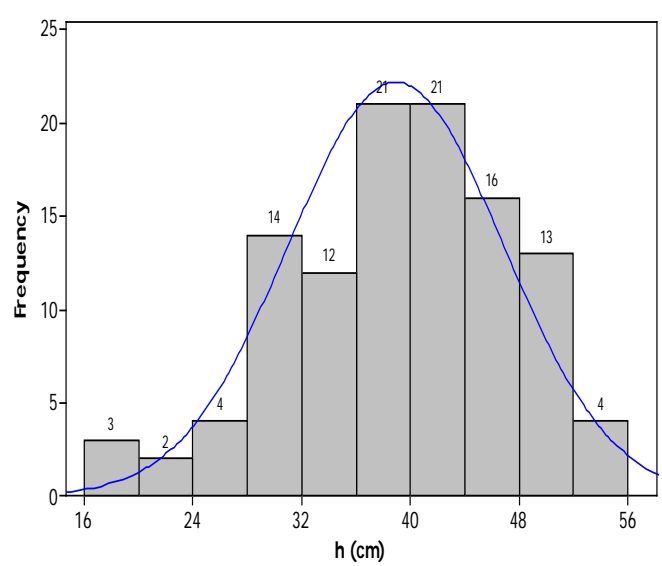

-C

Figure 6. -a: Identification of the stones inside Wall 1. Distribution of length (-b) and height $(-c)$ of the stones. 

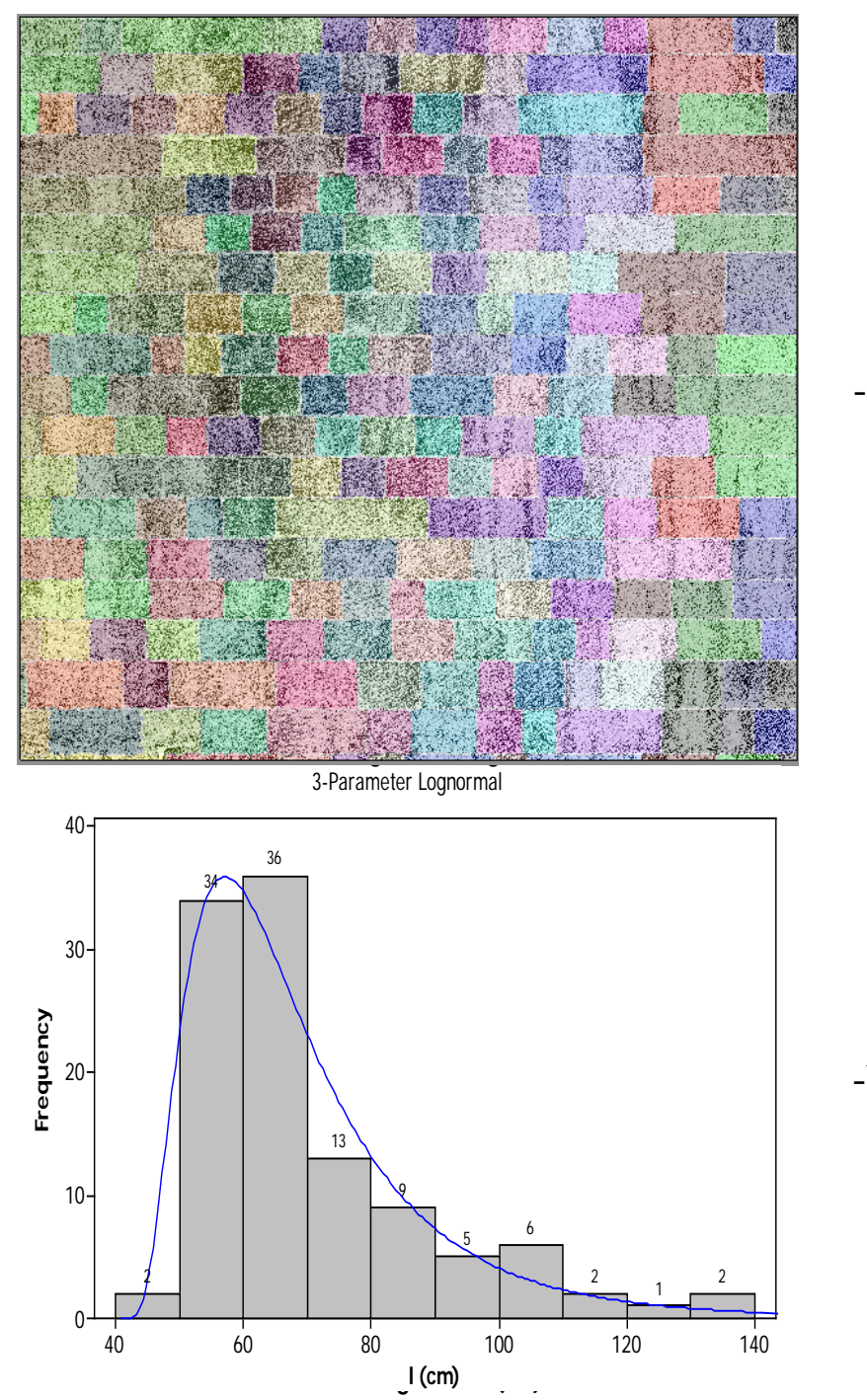

$-b$

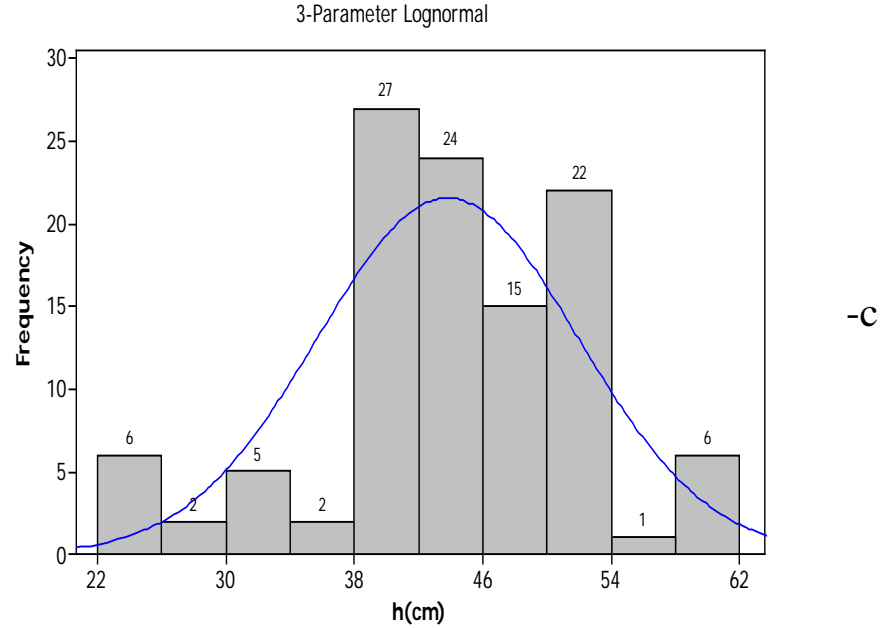

Figure 7. -a: Identification of the stones inside Wall 2.

Distribution of length (-b) and height (-c) of the stones. 


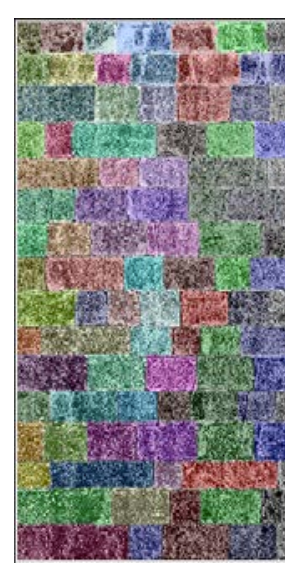

-a

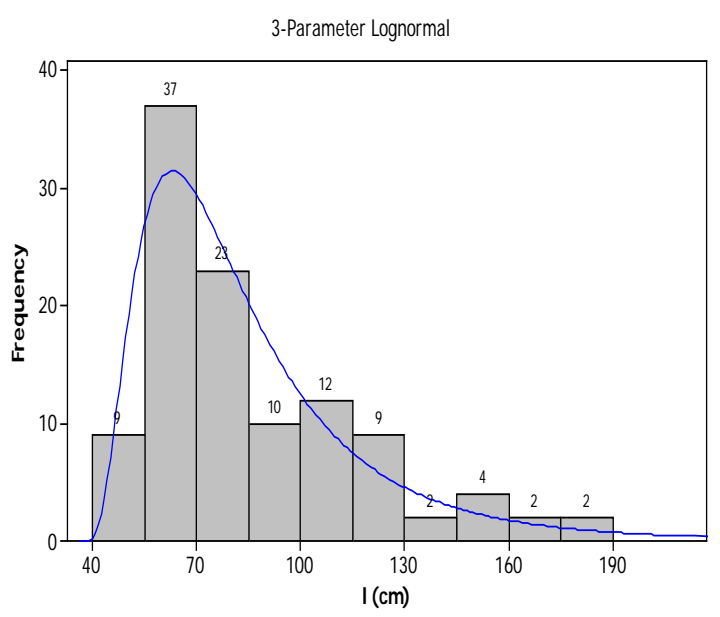

-b

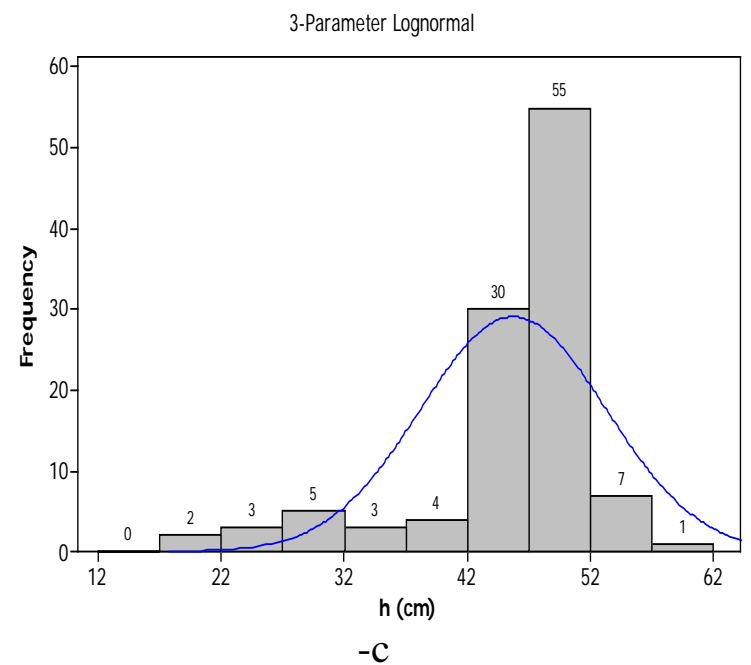

Figure 8. -a: Identification of the stones inside the Tower Wall. Distribution of length (-b) and height (-c) of the stones.

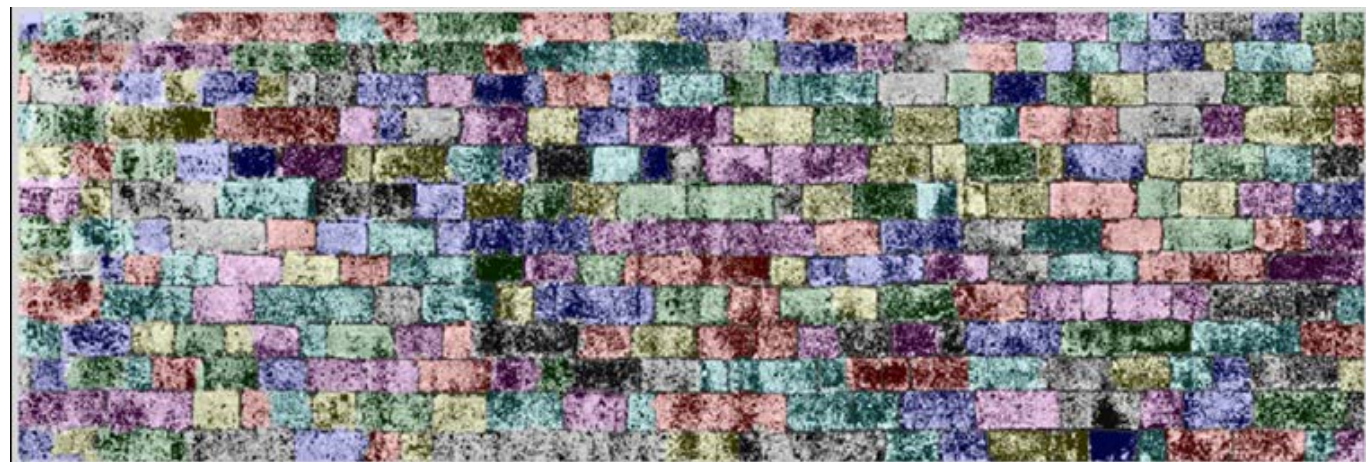

3-Parameter Lognormal

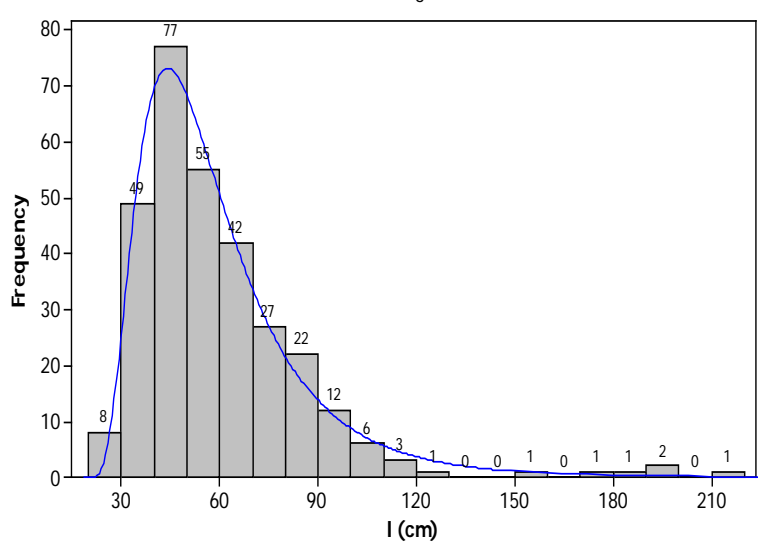

$-b$ $-\mathrm{a}$

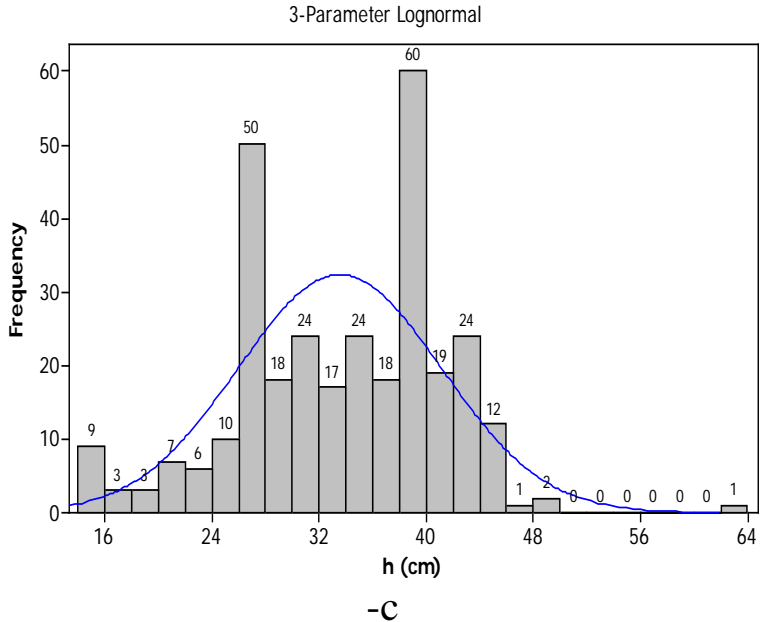

Figure 9. -a: Identification of the stones inside the Alcaçova Wall. Distribution of length (-b) and height (-c) of the stones. 

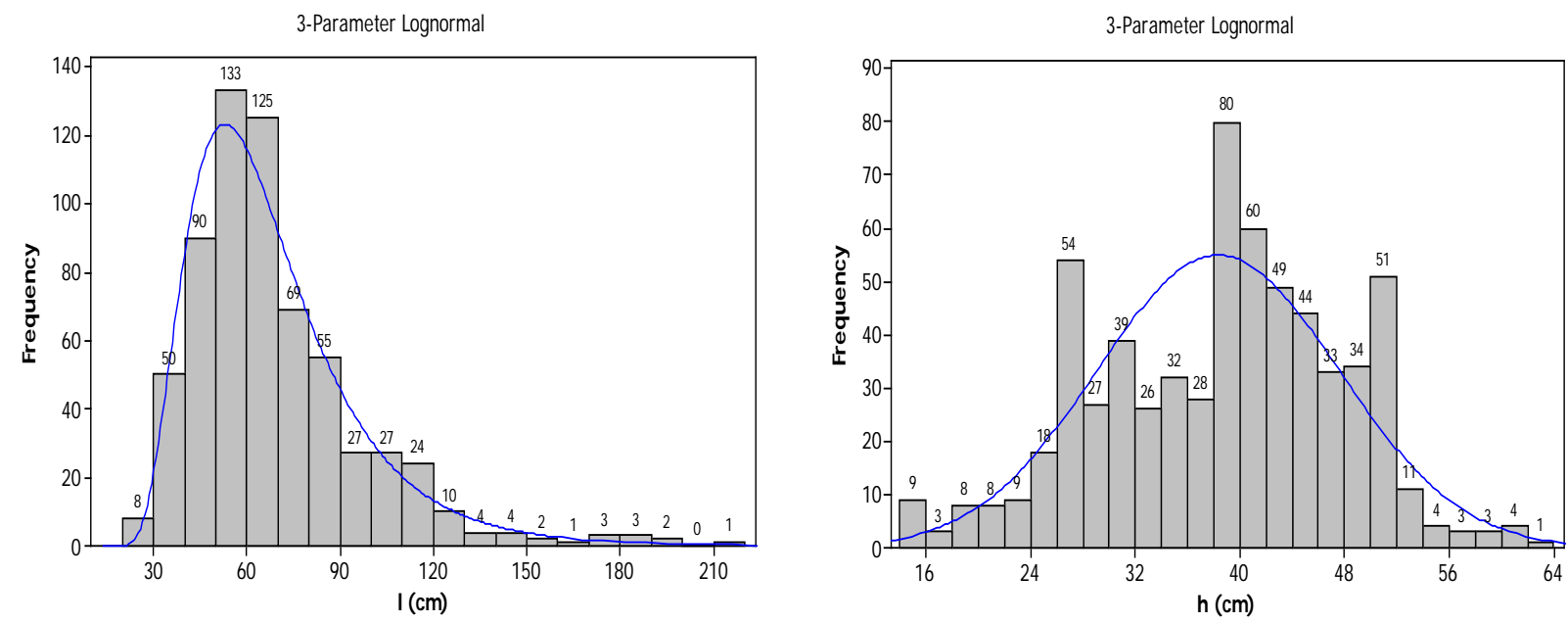

Figure 10. Distribution of length (l) and the height (h) of the stones in the all samples together. 

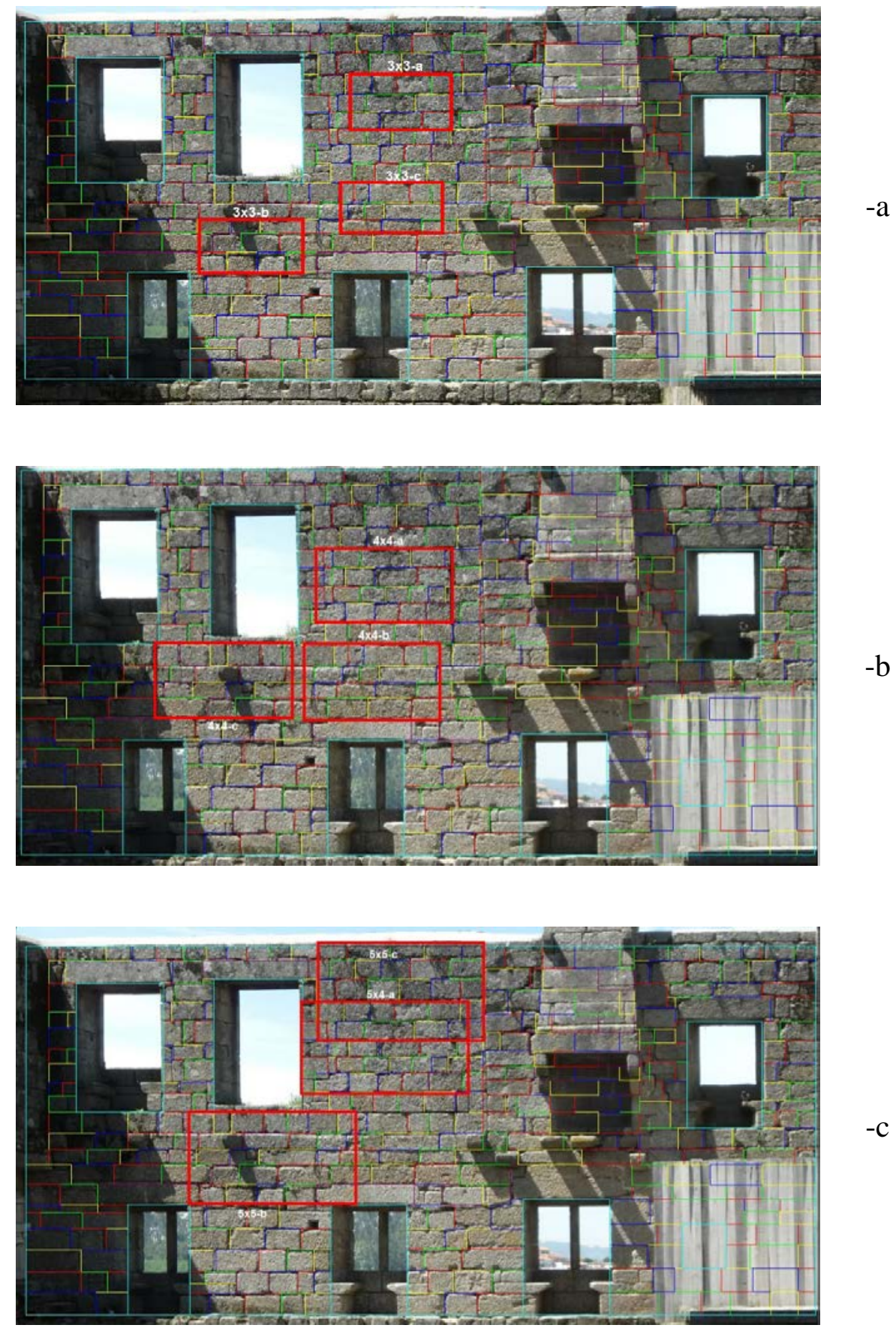

Figure 11 Location of representative volume elements. -a: 3x3 RVEs. -b: 4x4 RVEs. -c: 5x5 RVEs. 


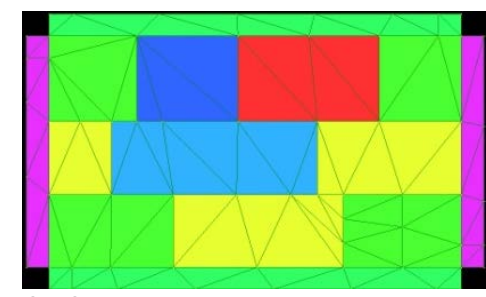

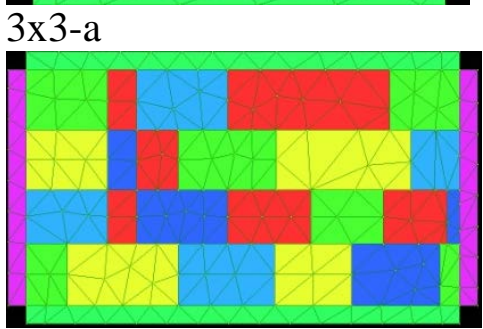

$4 \times 4-a$

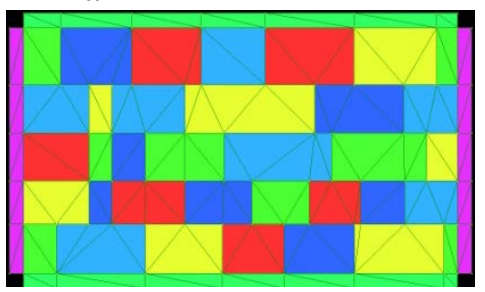

$5 \times 5-a$

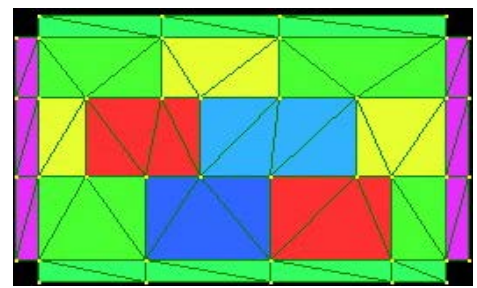

$3 \times 3-b$

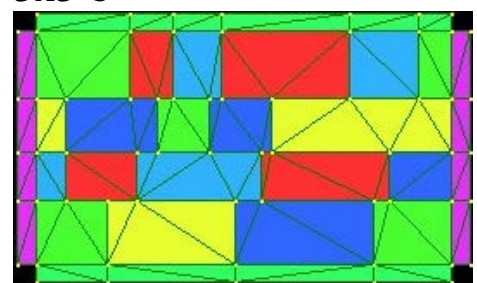

4x4-b

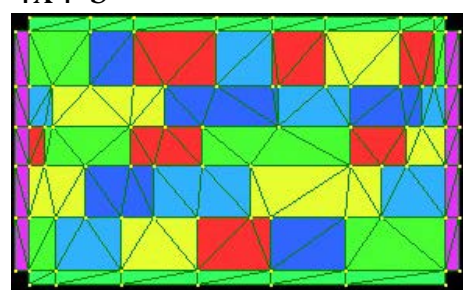

$5 \mathrm{x} 5-\mathrm{b}$

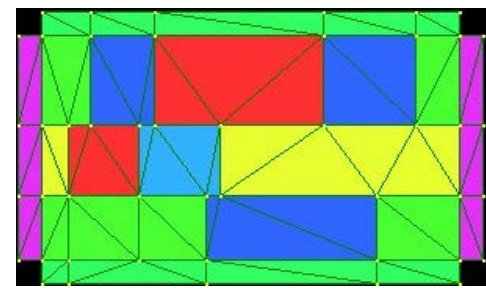

$3 \times 3-c$

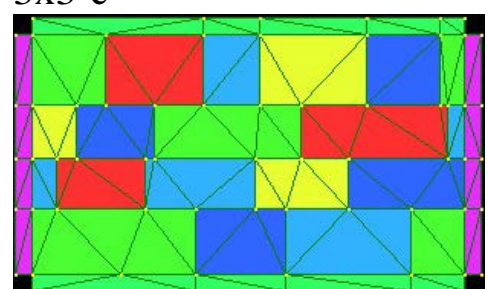

4x4-C

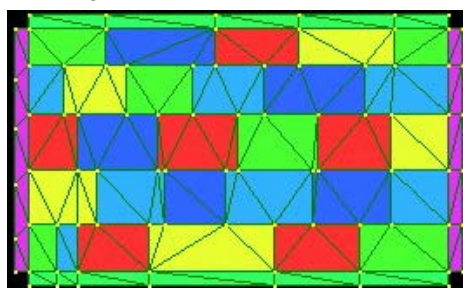

$5 \times 5-\mathrm{c}$

Figure 12. 3x3, 4x4 and 5x5 RVE numerical models, taken from Alcaçova wall with a triangular mesh

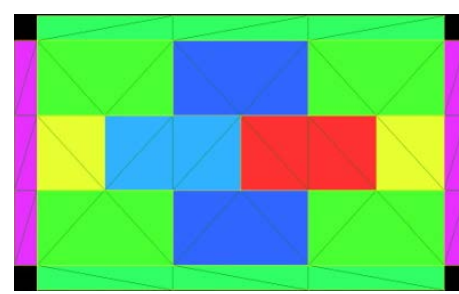

3x3-a

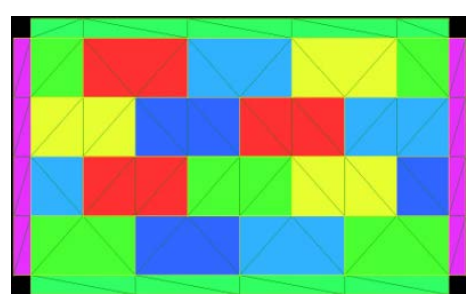

$4 \mathrm{x} 4-\mathrm{b}$

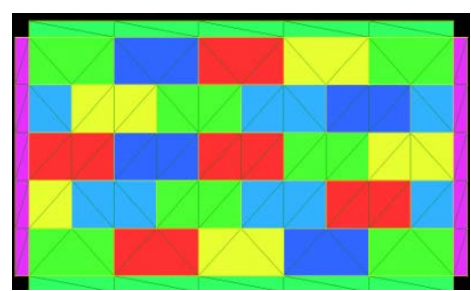

$5 \times 5-c$

Figure 13. Artificial RVE numerical model, taken from Alcaçova wall with a triangular mesh and periodic arrangement. 

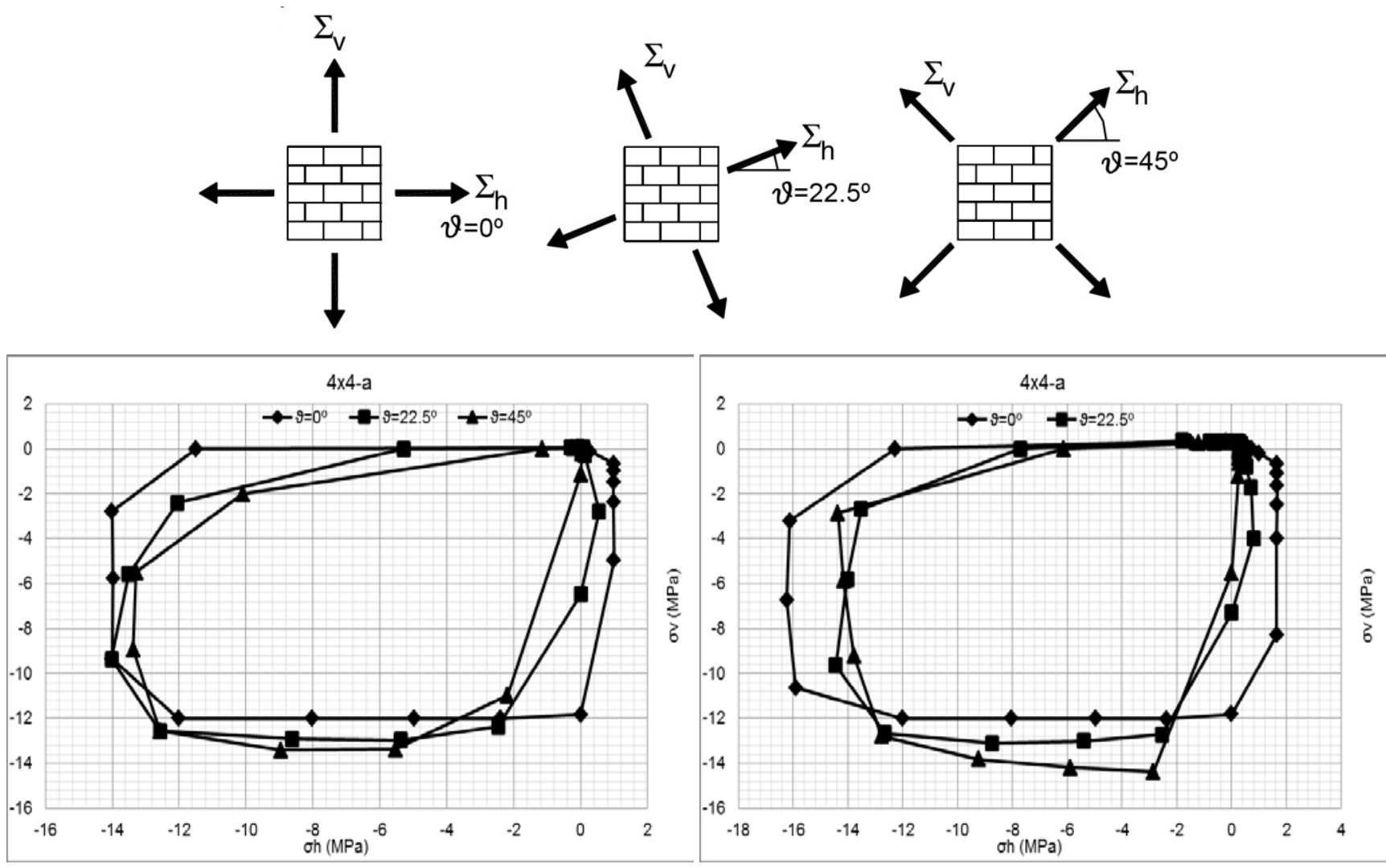

$-\mathrm{a}$

-b

Figure 14. Typical in-plane homogenized failure surfaces for a 4x4 RVE at different orientations of the principal stress axes with respect to bed joint direction. -a: weak mortar joint. -b: strong mortar. 

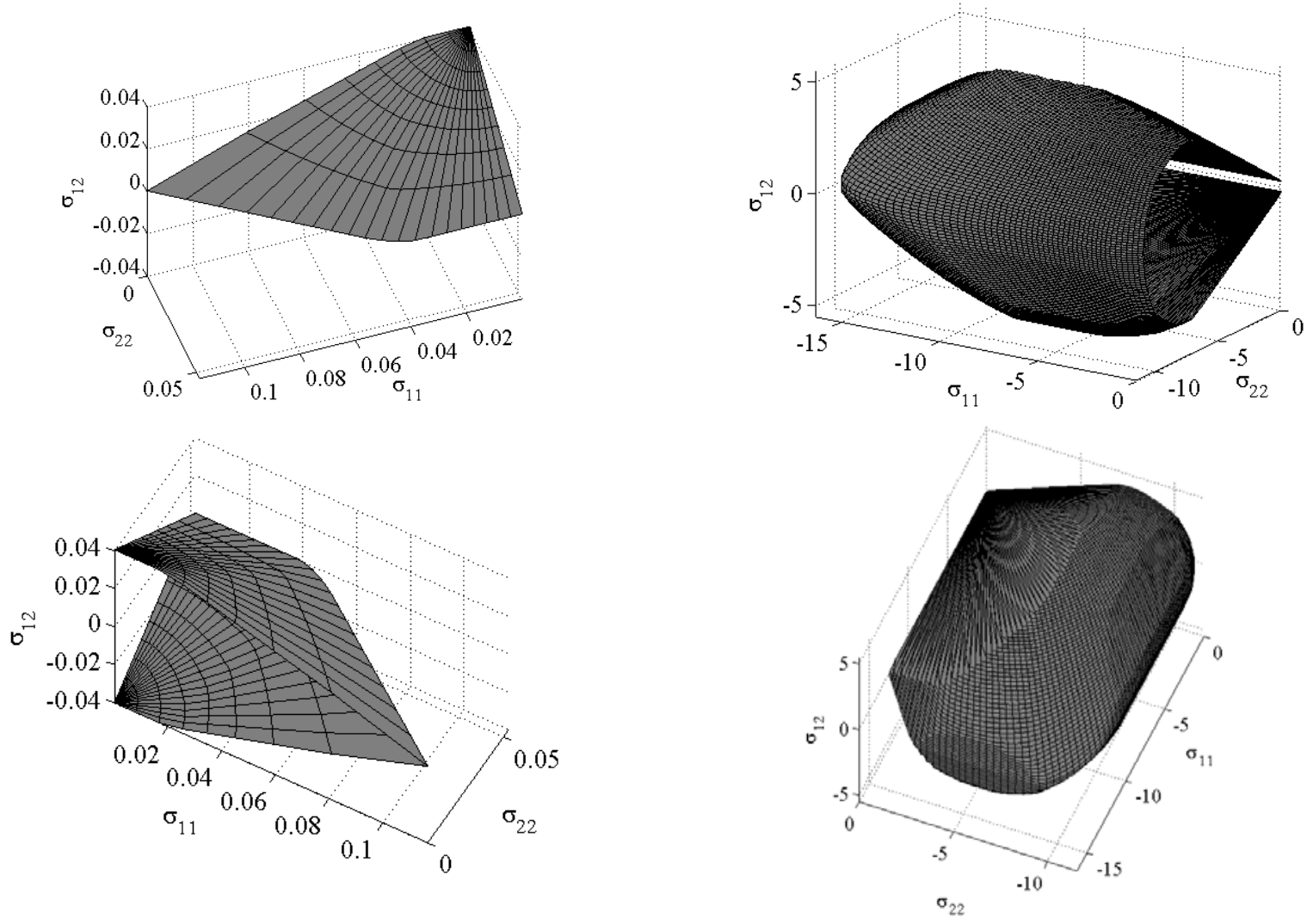

$-\mathrm{a}$

$-b$

Figure 15. 3D representation of the in-plane failure surface obtained for a $4 \times 4$ RVE in presence of weak mortar. (-a) tension-tension region (-b) compression-compression region. 


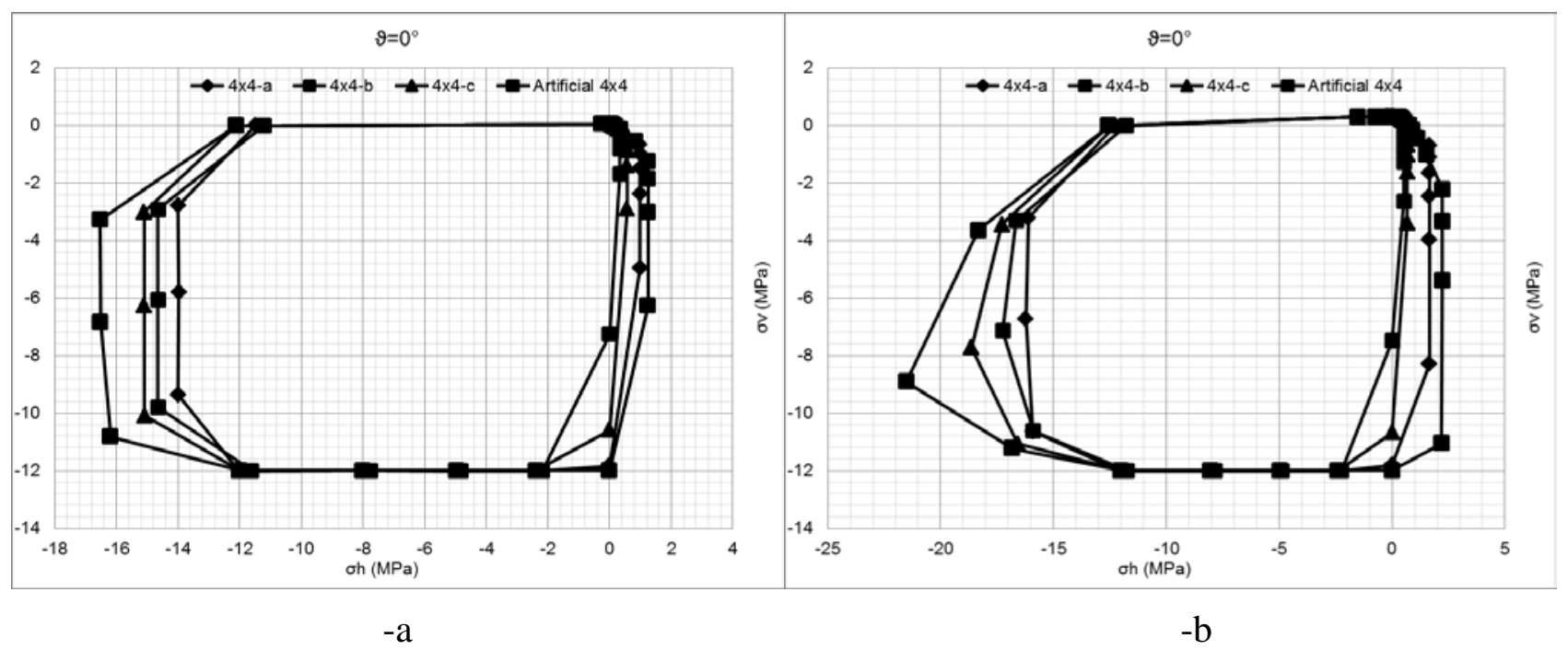

Figure 16. Comparison between in-plane homogenized failure surfaces obtained from $4 \times 4$ RVEs and artificial 4x4 RVEs at different orientations of the principal stress axes with respect to bed joint direction. -a: weak mortar joint. -b: strong mortar. 


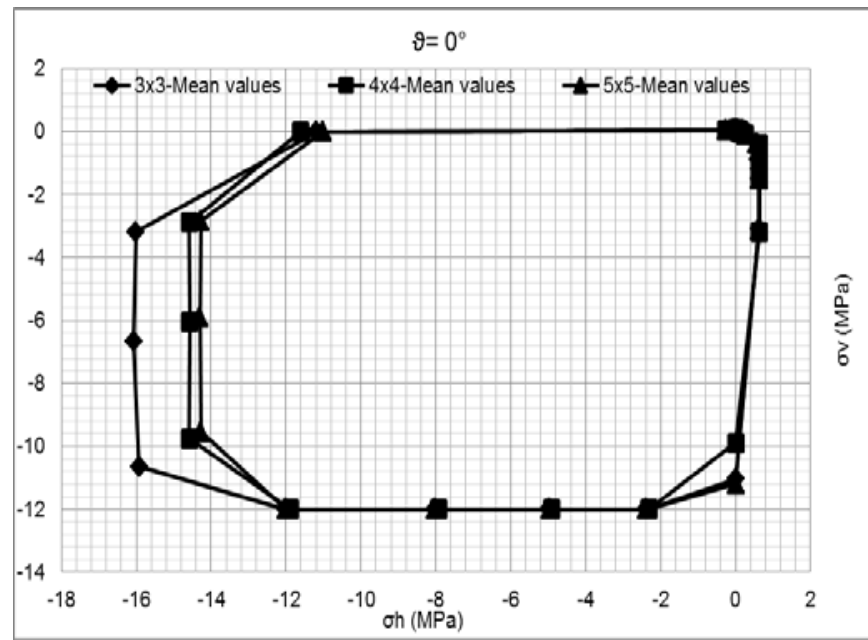

$-\mathrm{a}$

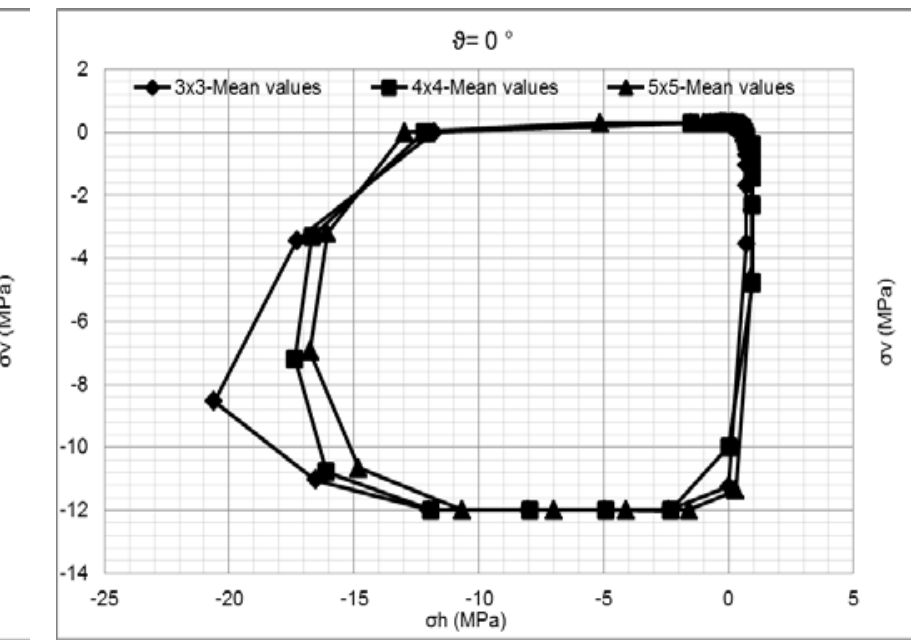

$-b$

Figure 17. Comparison between the mean values of in-plane homogenized failure surfaces for each size of RVEs with $\vartheta=0^{\circ}$. -a: weak mortar joint. -b: strong mortar.

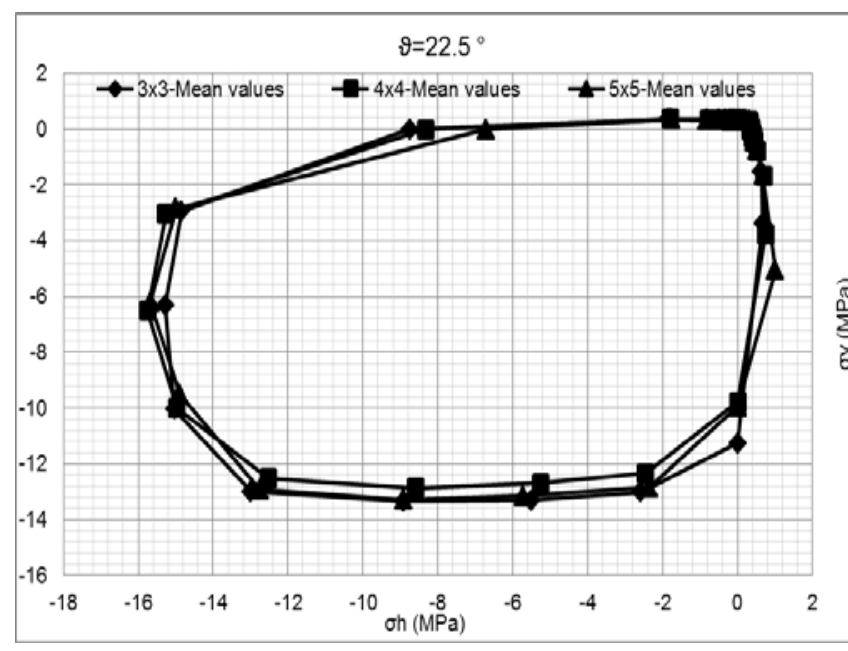

$-\mathrm{a}$

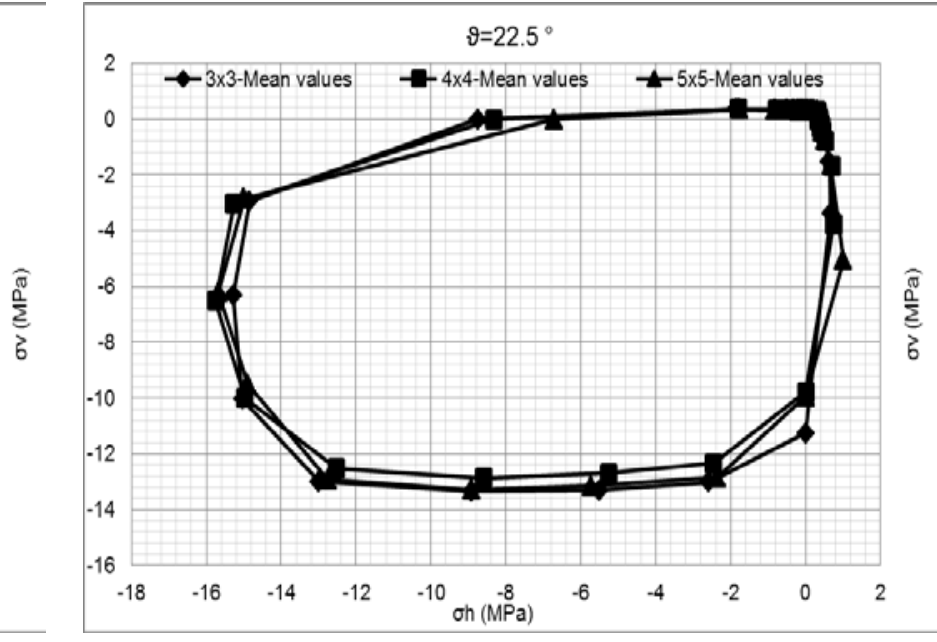

$-b$

Figure 18. Comparison between the mean values of in-plane homogenized failure surfaces for each size of RVEs with $\vartheta=22.5^{\circ}-\mathrm{a}$ : weak mortar joint. -b: strong mortar. 


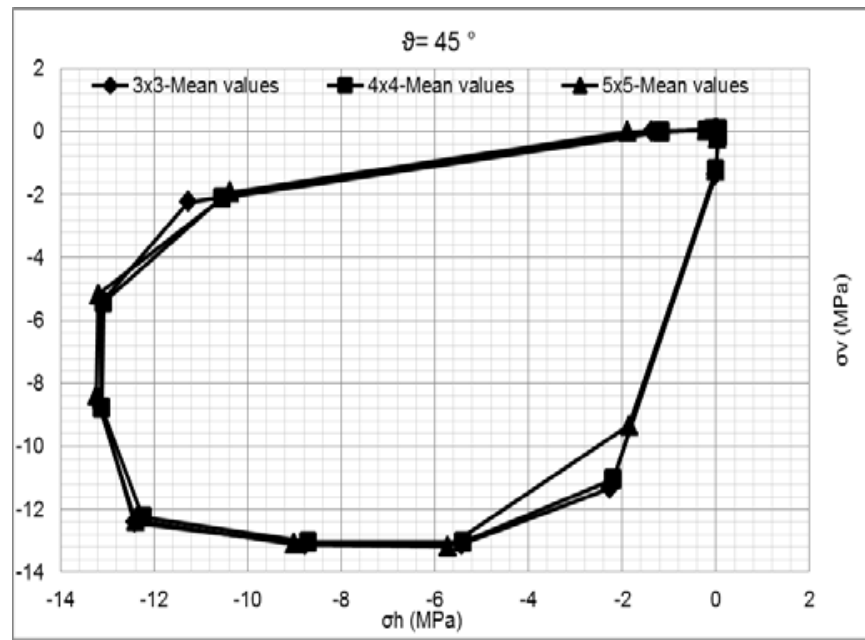

$-\mathrm{a}$

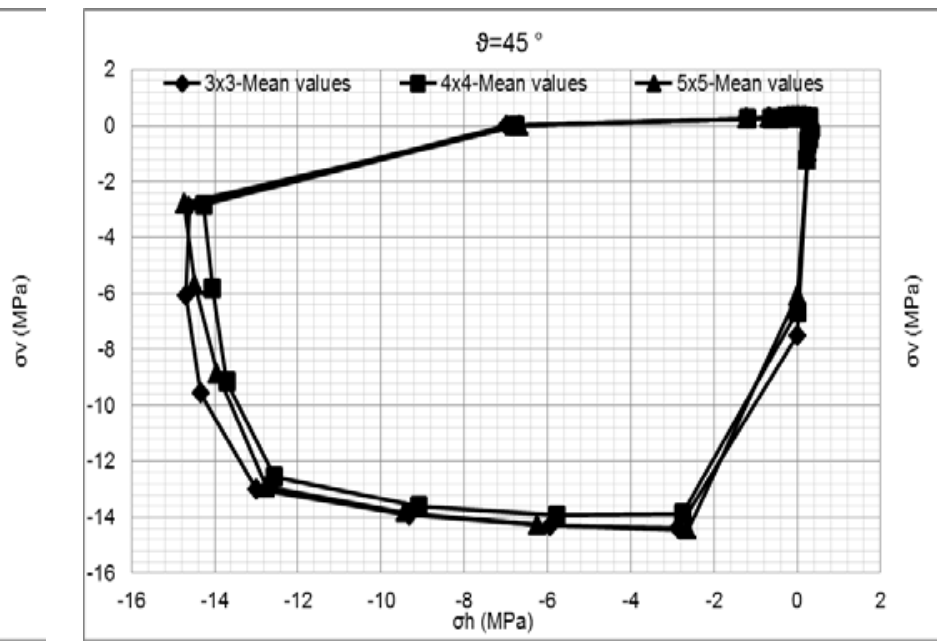

$-b$

Figure 19. Comparison between the mean values of in-plane homogenized failure surfaces for each size of RVEs with $\vartheta=45^{\circ}$. $-\mathrm{a}$ : weak mortar joint. -b: strong mortar. 

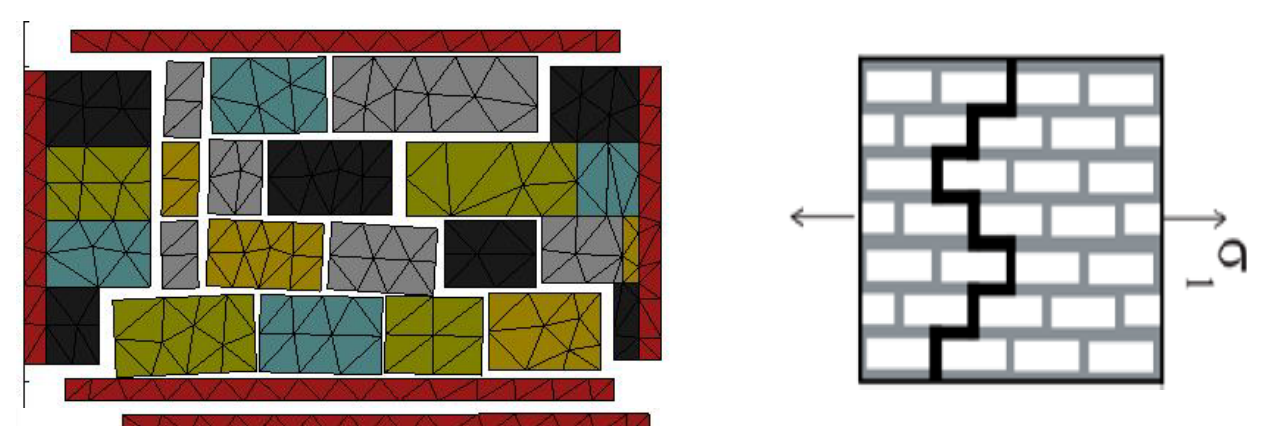

$-a$

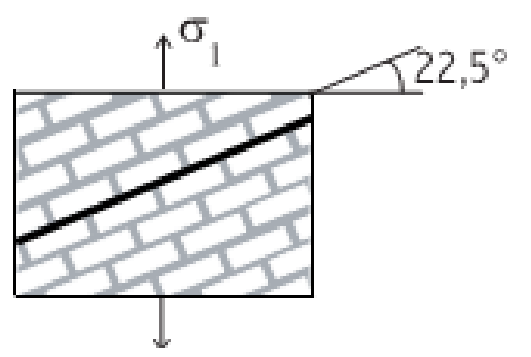

-b
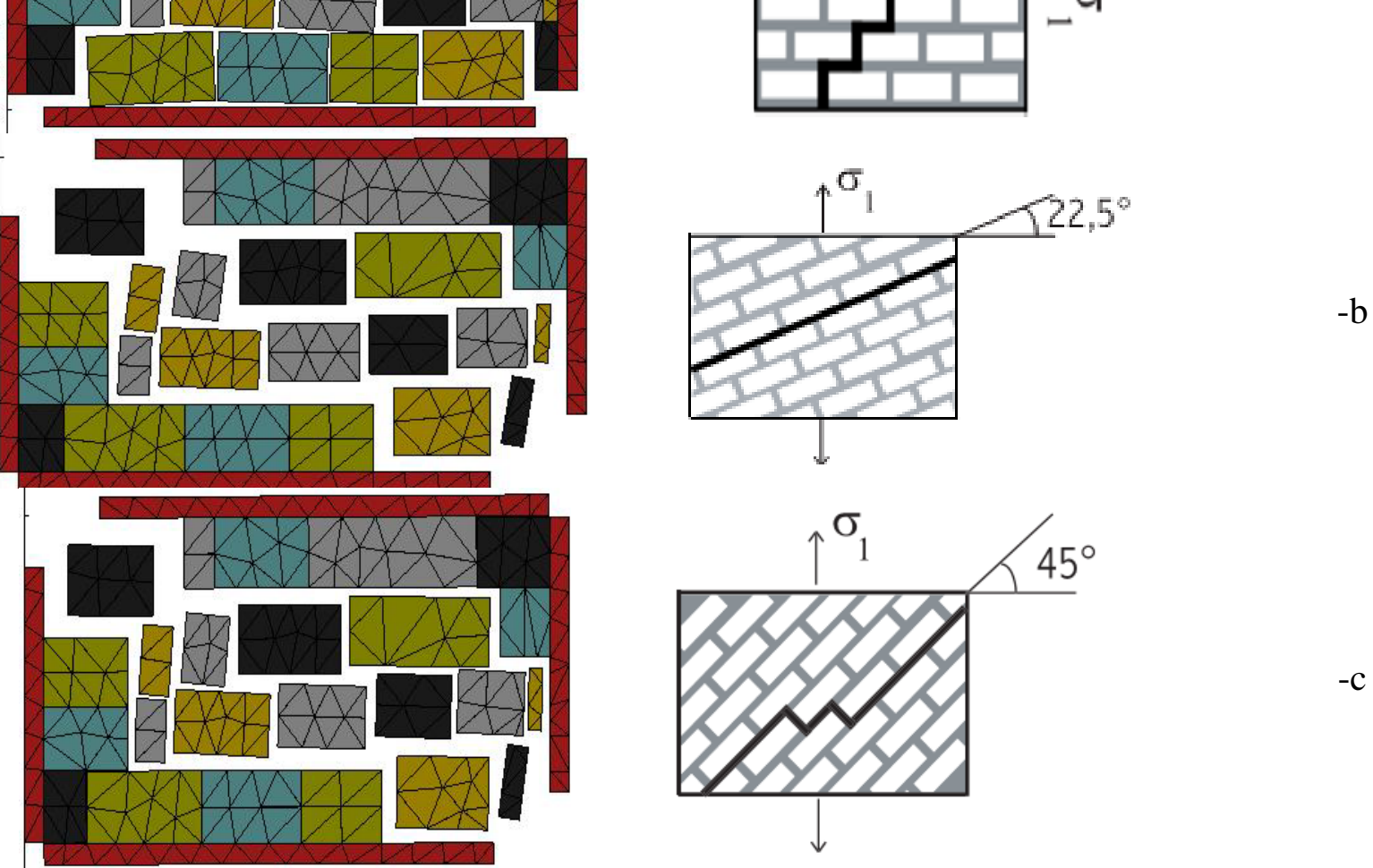

Figure 20. Qualitative comparison of mode failure between 4x4 RVE from masonry with strong mortar at orientations of the load equal to $\vartheta=0,22.5^{\circ}$ and $45^{\circ}$ (respectively subfigures $-\mathrm{a},-\mathrm{b}$ and $-\mathrm{c}$ ) and experimentally observed failure modes 

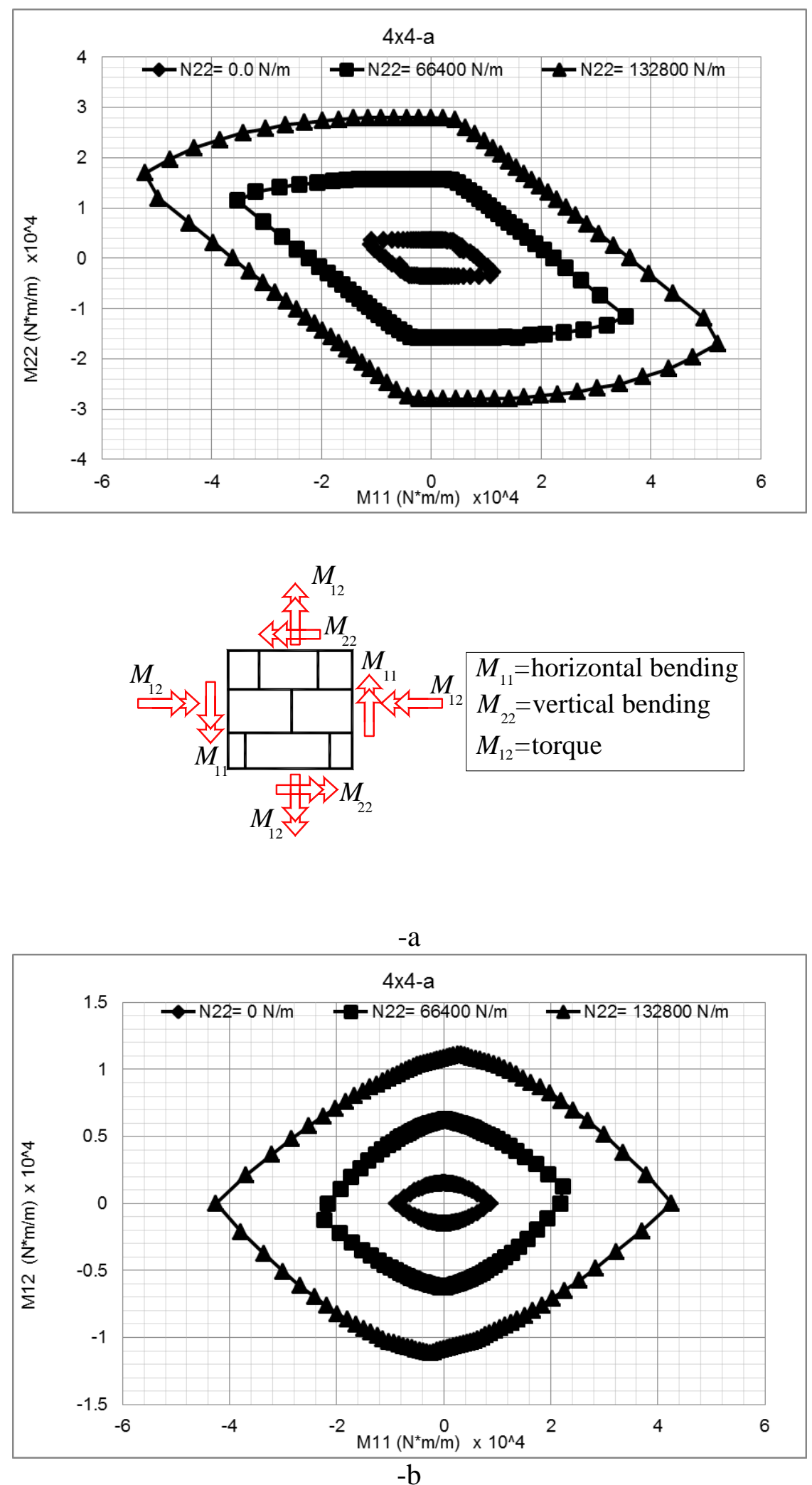

Figure 21. Out-of-plane homogenized failure surfaces (-a: M11-M22, -b: M11-M12) for 4x4 RVE of masonry with weak mortar at different vertical compressive loads. 

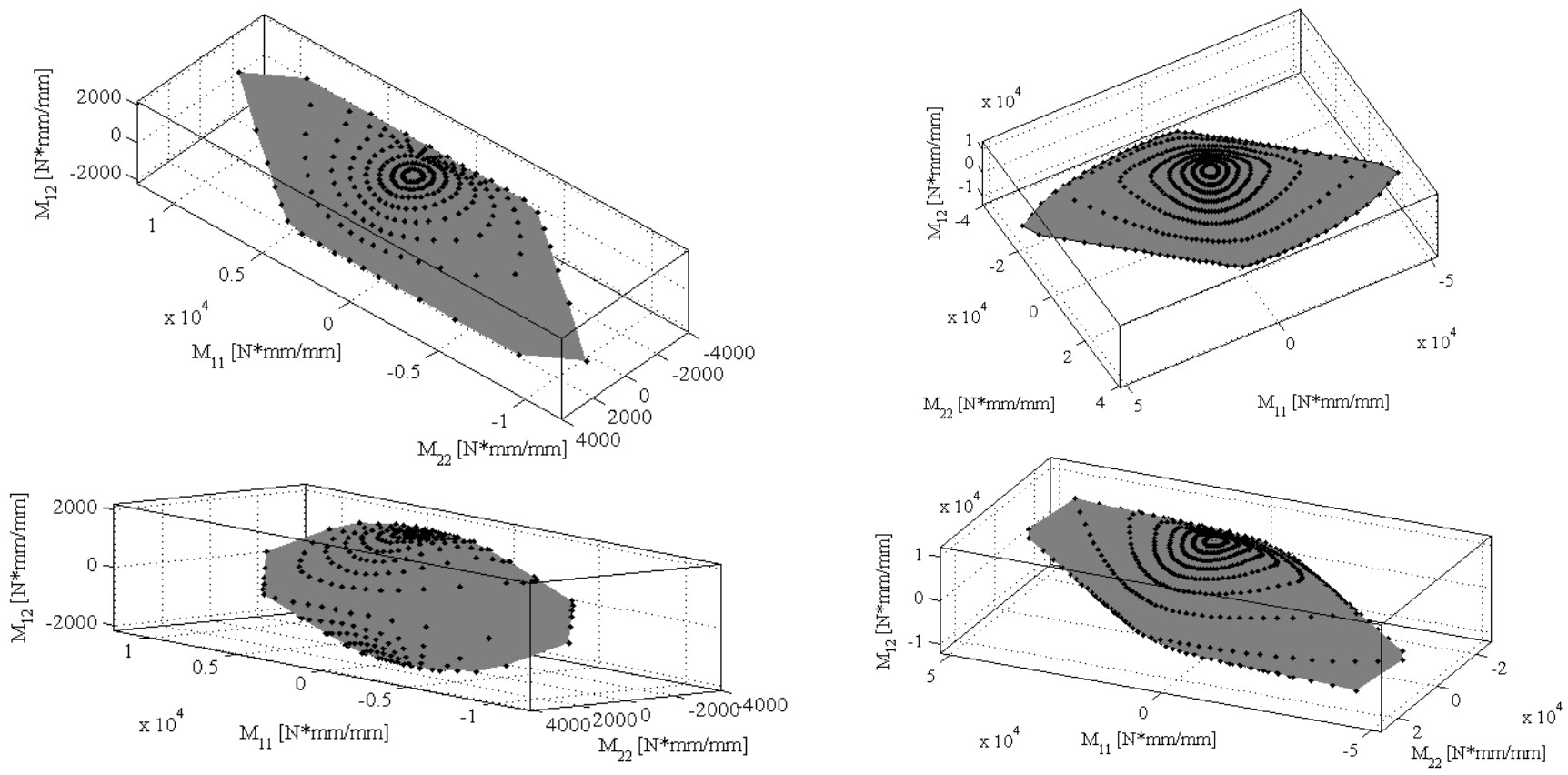

$-\mathrm{a}$

$-b$

Figure 22 3D representation of the out-of-plane failure surface obtained for a 4x4 RVE in presence of weak mortar. (-a) wall head (-b) wall foot (maximum vertical membrane compression). 

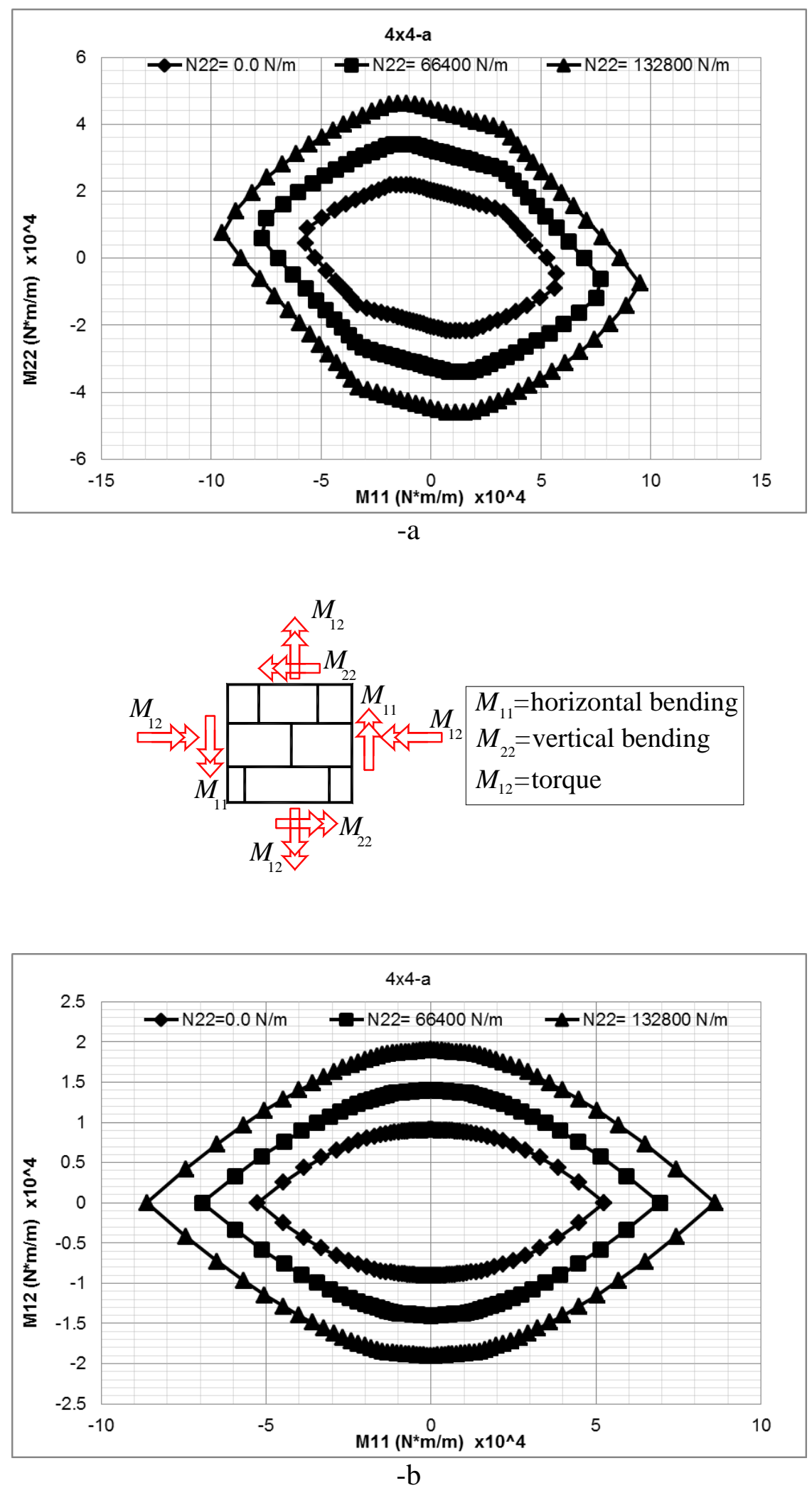

Figure 23. Out-of-plane homogenized failure surfaces (-a: M11-M22, -b: M11-M12) for 4x4 RVE of masonry with strong mortar at different vertical compressive loads. 

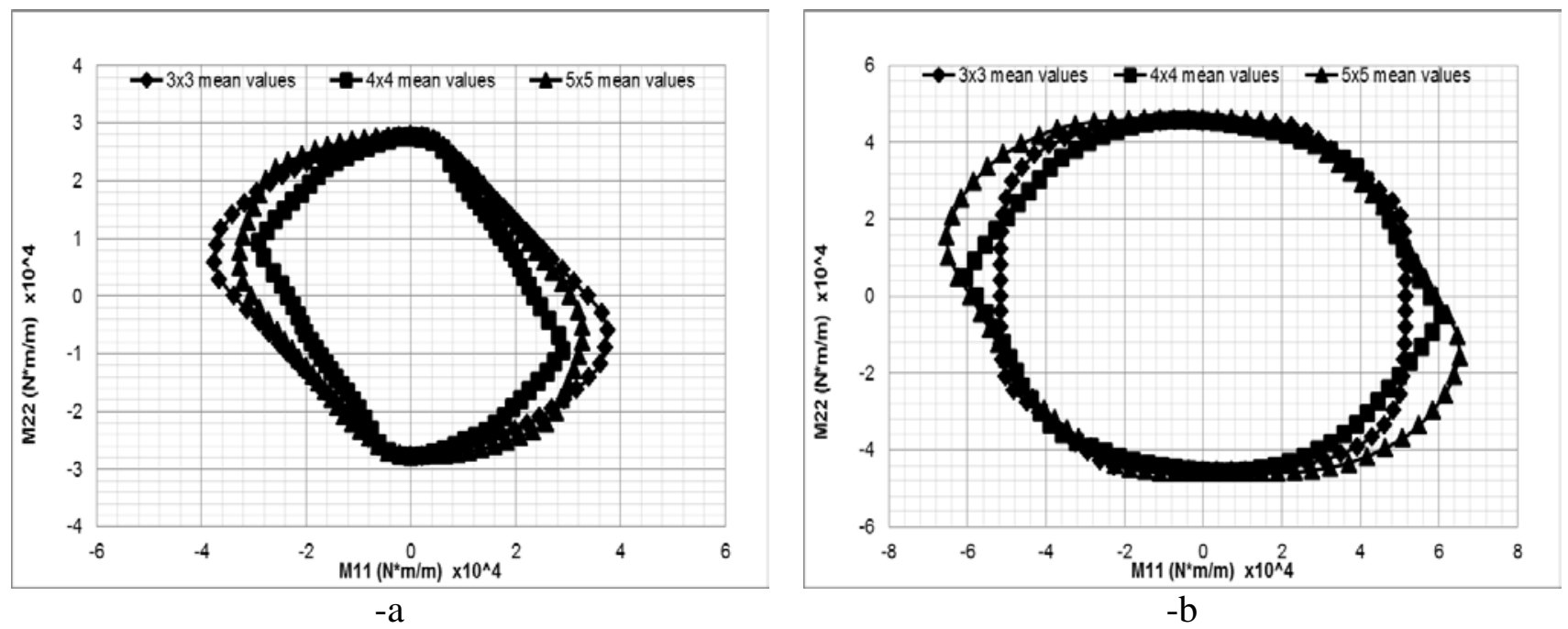

Figure 24. Comparison between the mean values of out-of-plane homogenized failure surfaces for each RVE size. -a: RVE with weak mortar. -b: RVE with strong mortar
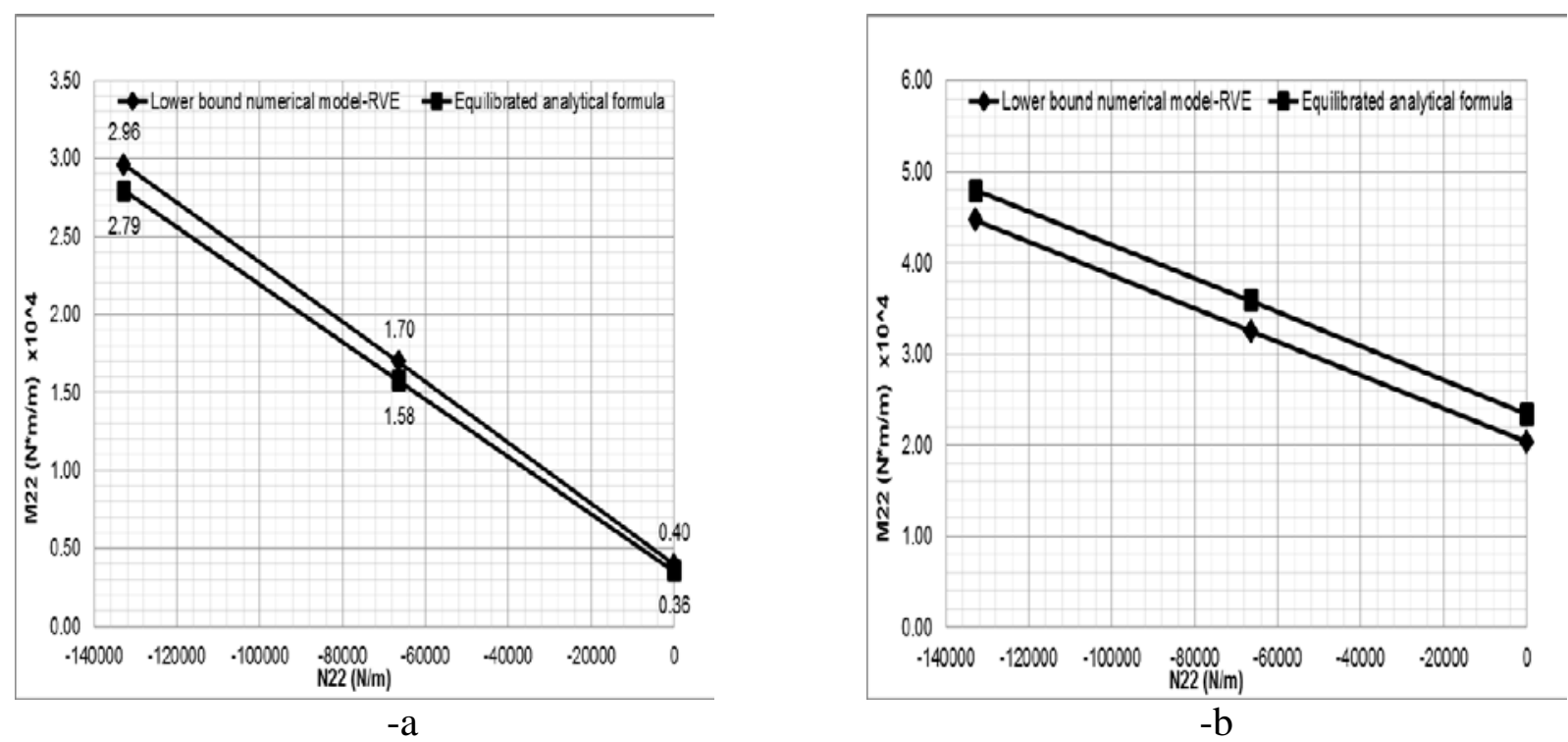

Figure 25. Comparison between RVEs and a simplified analytical formula performed on bed joint to predict vertical ultimate bending moment with increasing vertical load: -a: RVE with weak mortar. -b: RVE with strong mortar 

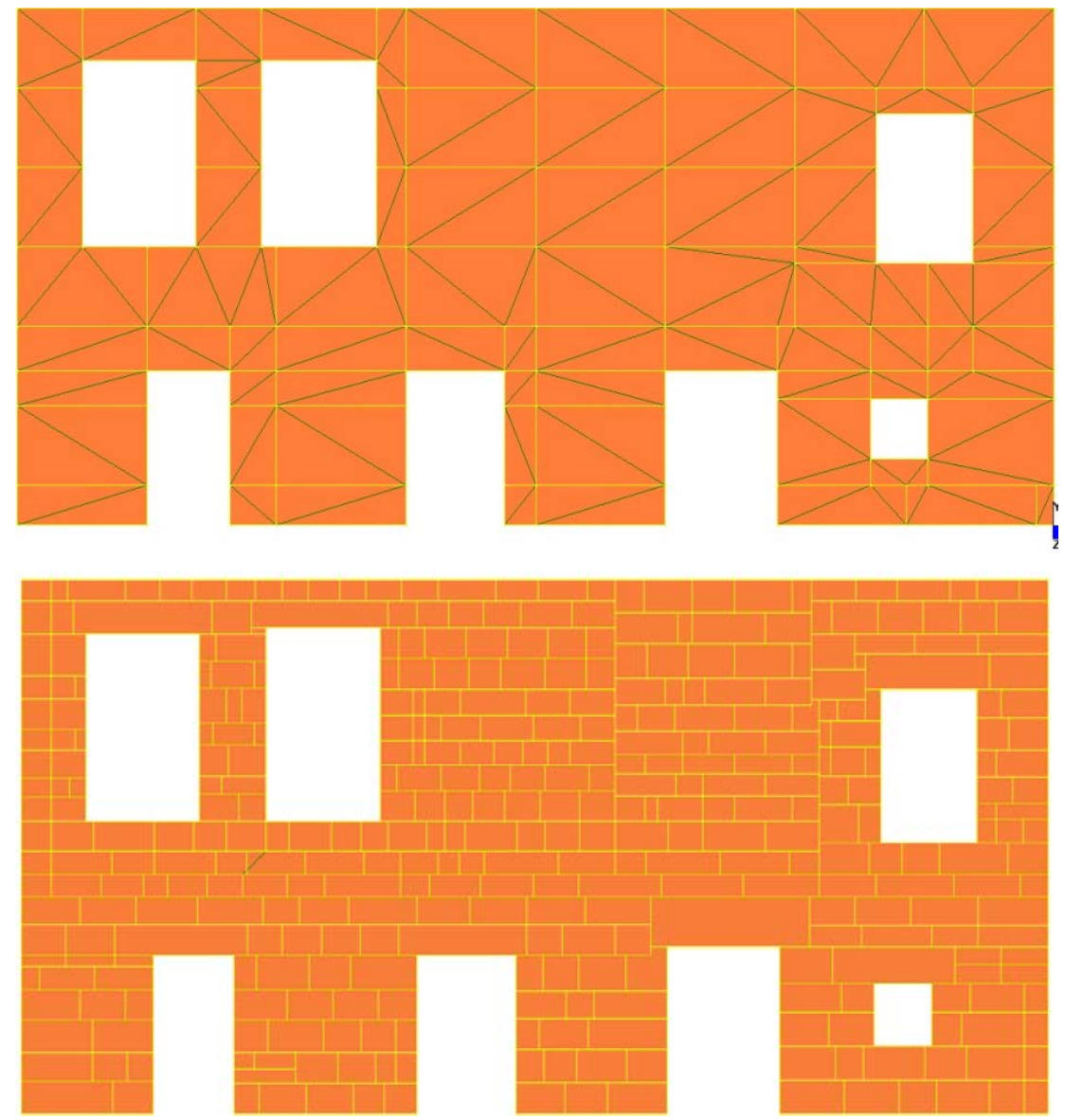

Figure 26. Numerical homogenous (top, 173 elements) and heterogeneous (bottom, 1299 elements) models of Alcaçova wall. 


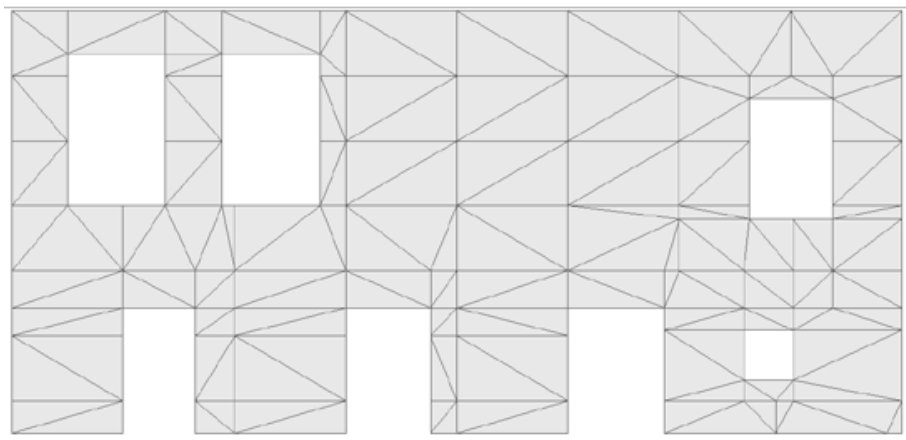

Mesh C 173 elements

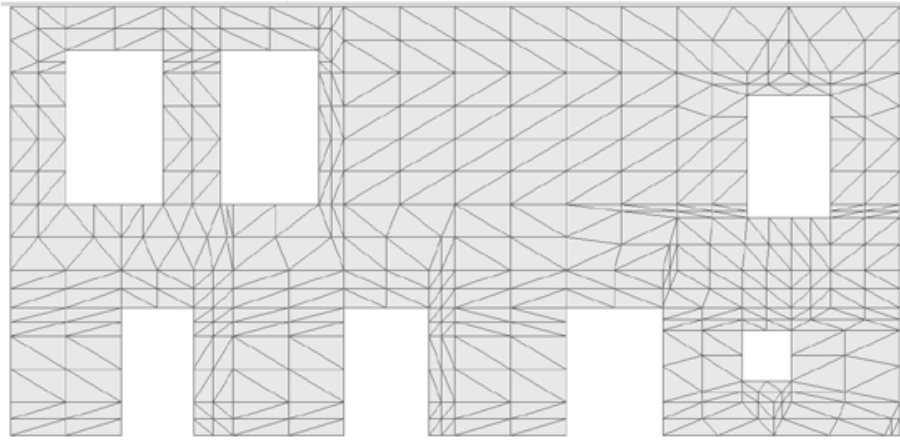

Mesh B 692 elements

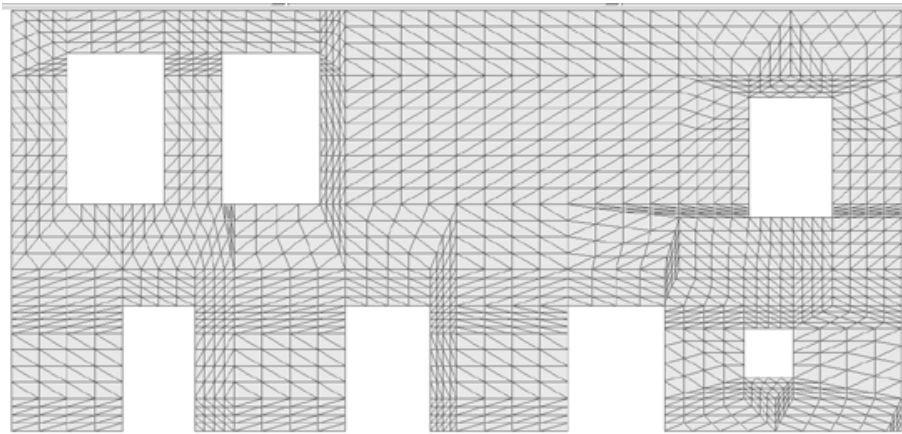

Mesh C 2768 elements

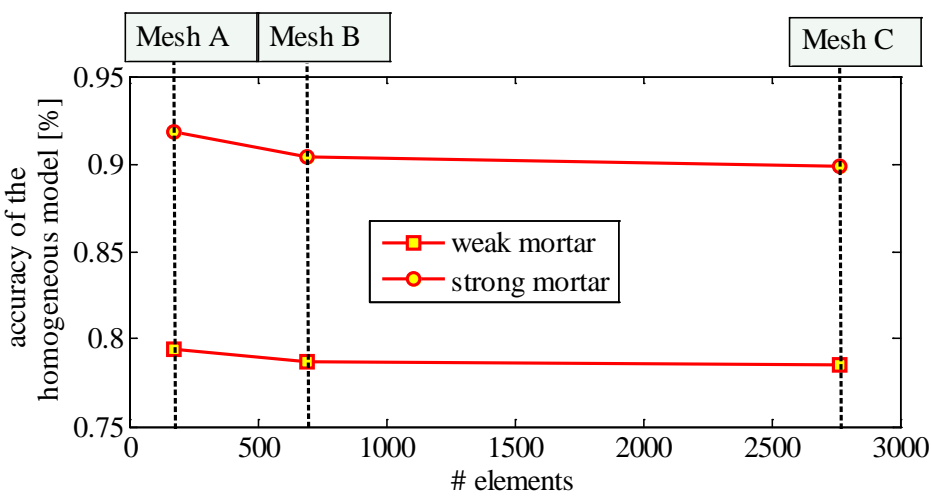

Figure 27 . Mesh sensitivity analysis conducted on three discretizations with increasing number of elements. 

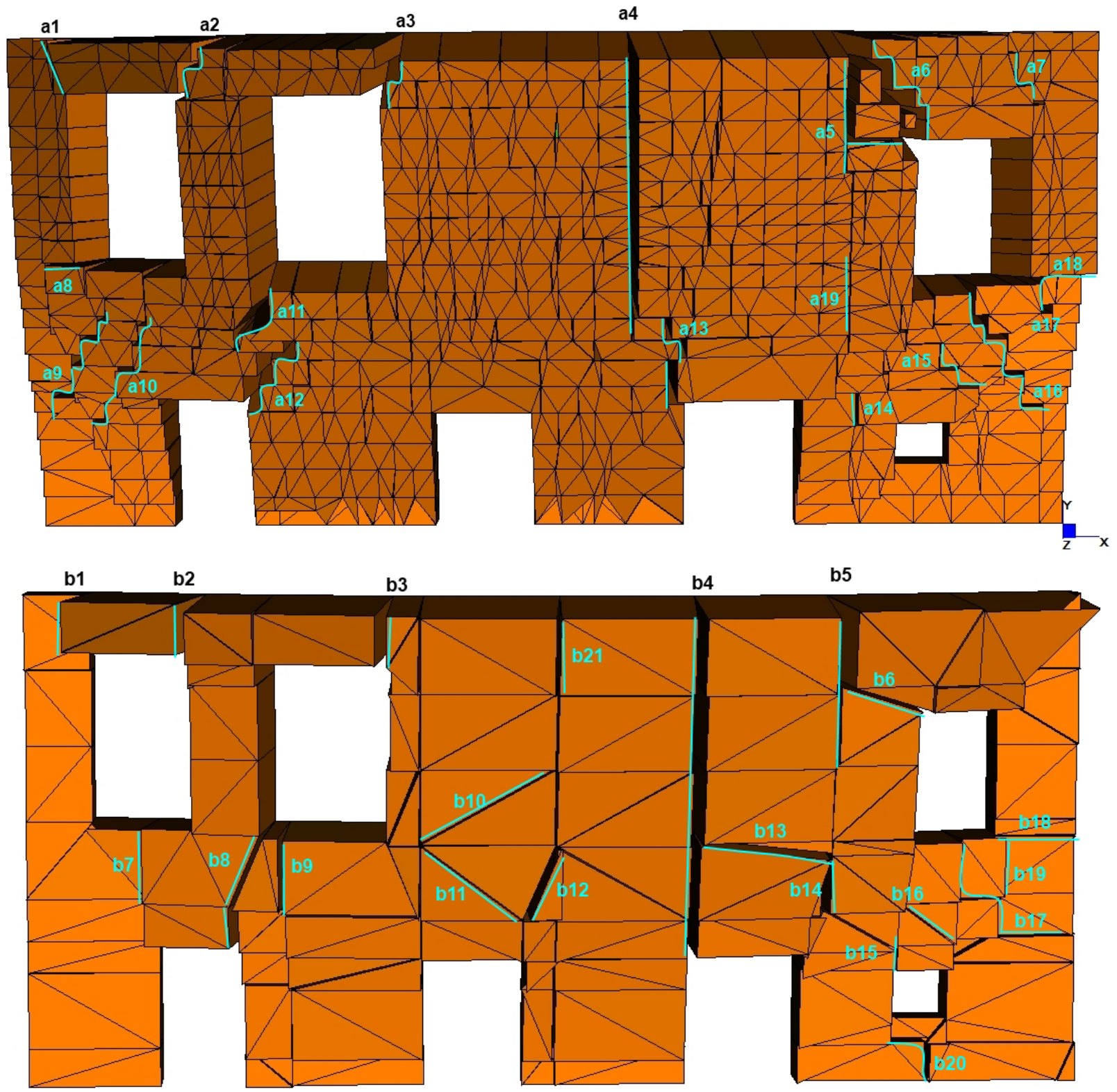

Figure 28. Collapse mechanism of the heterogeneous (top) and homogeneous (bottom) model with weak mortar. 


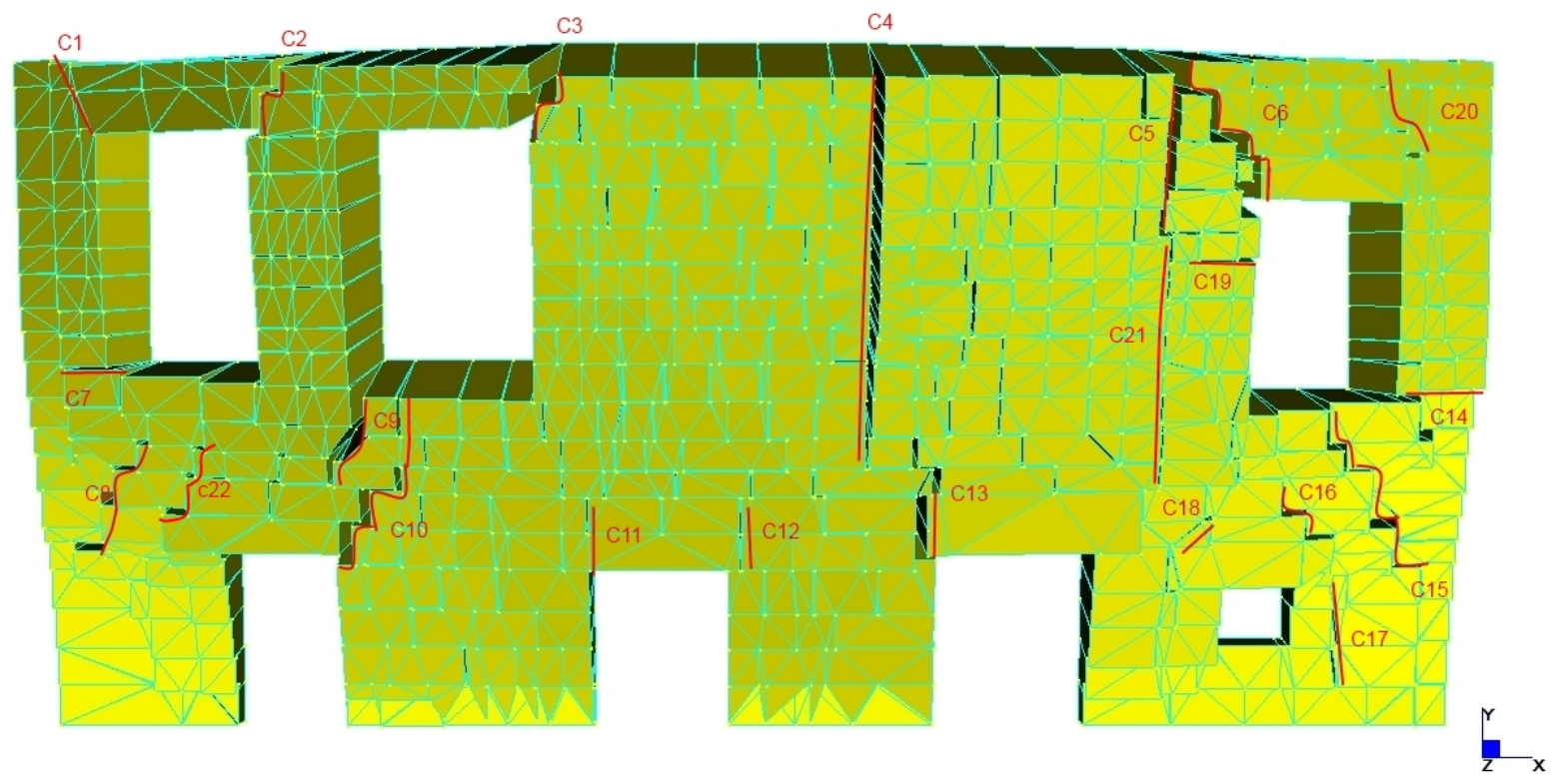

d1 $\quad$ d2

d5 $\quad$ d6

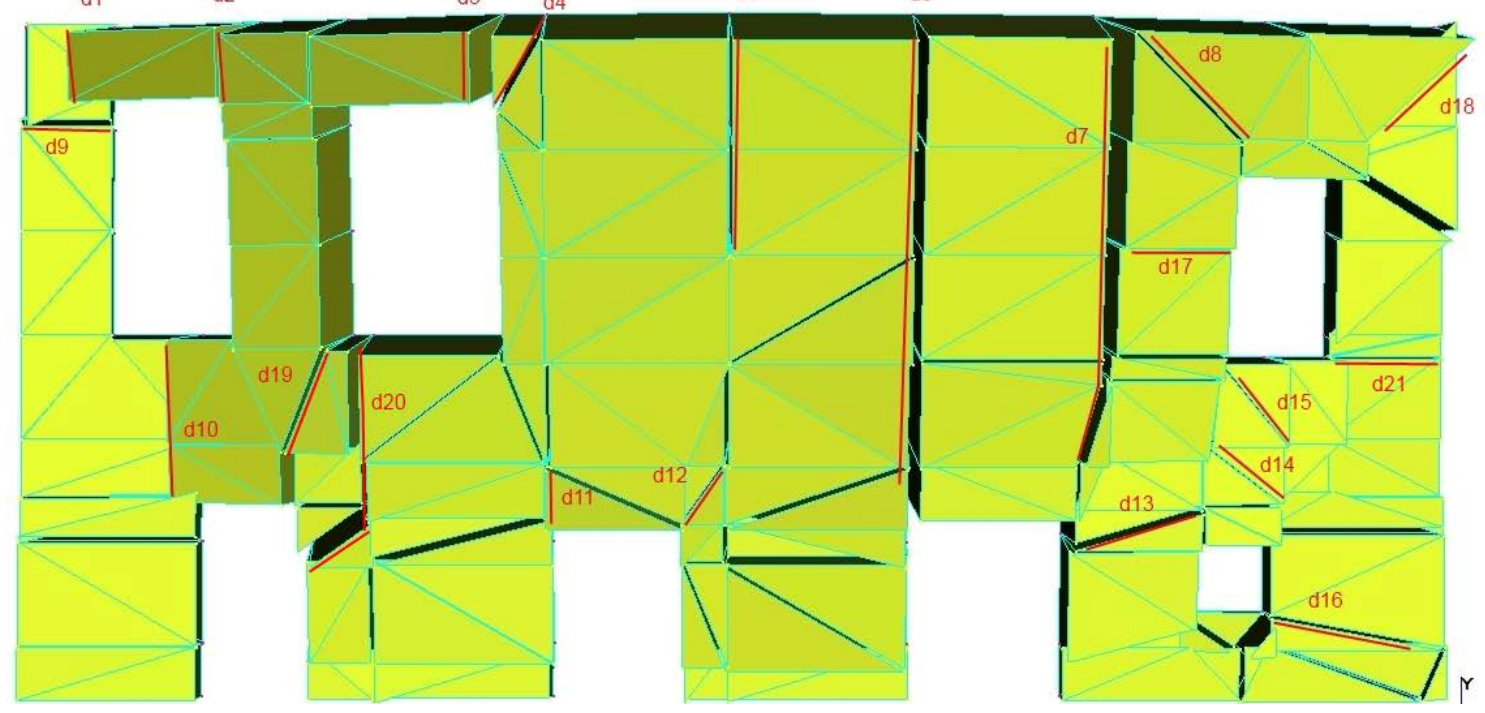

Figure 29. Collapse mechanism of the heterogeneous (top) and homogeneous (bottom) model with strong mortar. 


\begin{tabular}{|l|l|c|c|}
\hline \multicolumn{2}{|c|}{} & $\begin{array}{c}\text { Laser } \\
\text { scanning }\end{array}$ & Photography \\
\hline \multirow{2}{*}{ Length } & Average [cm] & 61 & 60 \\
\cline { 2 - 4 } & CoV & $51 \%$ & $44 \%$ \\
\hline \multirow{2}{*}{ Height } & Average [cm] & 41 & 34 \\
\cline { 2 - 4 } & CoV & $9 \%$ & $23 \%$ \\
\hline
\end{tabular}

Table 1. Laser scanning vs photography results for Alcáçova wall

\begin{tabular}{|c|c|c|c|c|c|c|c|c|}
\hline \multirow{2}{*}{ Wall } & \multirow[b]{2}{*}{$\begin{array}{c}\text { Number of } \\
\text { units }\end{array}$} & \multirow{2}{*}{\begin{tabular}{|c} 
Ratio \\
Height \\
$/$ \\
Length \\
$h / l$ \\
\end{tabular}} & \multicolumn{3}{|c|}{ Length } & \multicolumn{3}{|c|}{ Height } \\
\hline & & & $\begin{array}{c}\text { Average } \\
\text { [cm] } \\
(\mathrm{CoV}) \\
\end{array}$ & $\begin{array}{l}\text { Typical [cm] } \\
\text { (Frequency) }\end{array}$ & $\begin{array}{l}\text { Range } \\
{[\mathrm{cm}]}\end{array}$ & $\begin{array}{l}\text { Height }[\mathrm{cm}] \\
(\mathrm{CoV})\end{array}$ & $\begin{array}{l}\text { Typical }[\mathrm{cm}] \\
\text { (Frequency) }\end{array}$ & $\begin{array}{l}\text { Range } \\
{[\mathrm{cm}]}\end{array}$ \\
\hline Wall 1 & 110 & 0.51 & $\begin{array}{c}76 \\
(34 \%)\end{array}$ & $\begin{array}{c}70 \\
(27 \%)\end{array}$ & $40-170$ & $\begin{array}{c}39 \\
(20 \%)\end{array}$ & $\begin{array}{c}40 \\
(38 \%)\end{array}$ & $20-40$ \\
\hline Wall 2 & 110 & 0.63 & $\begin{array}{c}70 \\
(27 \%)\end{array}$ & $\begin{array}{c}65 \\
(27 \%)\end{array}$ & $45-135$ & $\begin{array}{c}44 \\
(19 \%)\end{array}$ & $\begin{array}{c}40 \\
(25 \%)\end{array}$ & $25-60$ \\
\hline Tower & 110 & 0.54 & $\begin{array}{c}85 \\
(37 \%)\end{array}$ & $\begin{array}{c}65 \\
(34 \%)\end{array}$ & $50-180$ & $\begin{array}{c}46 \\
(17 \%)\end{array}$ & $\begin{array}{c}50 \\
(35 \%)\end{array}$ & $15-60$ \\
\hline Alcaçova & 308 & 0.56 & $\begin{array}{c}60 \\
(44 \%)\end{array}$ & $\begin{array}{c}45 \\
(25 \%)\end{array}$ & $25-210$ & $\begin{array}{c}34 \\
(23 \%)\end{array}$ & $\begin{array}{c}40 \\
(35 \%)\end{array}$ & $15-60$ \\
\hline $\begin{array}{c}\text { Full } \\
\text { Sample }\end{array}$ & $\begin{array}{l}\text { Weighted } \\
\text { average }\end{array}$ & 0.56 & $\begin{array}{c}69 \\
(40 \%)\end{array}$ & $\begin{array}{c}55 / 65 \\
(21 \%) /(20 \%)\end{array}$ & $25-210$ & $\begin{array}{c}38 \\
(24 \%)\end{array}$ & $\begin{array}{c}40 \\
(35 \%)\end{array}$ & $15-60$ \\
\hline
\end{tabular}

Table 2. Statistical parameters of wall samples from Guimarães castle 


\begin{tabular}{|c|c|c|}
\hline & Width $(\mathrm{cm})$ & Height $(\mathrm{cm})$ \\
\hline Mean Stone & 59.81 & 33.55 \\
\hline $3 \times 3$ & 179.43 & 100.65 \\
\hline $4 \times 4$. & 239.24 & 134.2 \\
\hline $5 \times 5$ & 299.05 & 167.75 \\
\hline
\end{tabular}

Table 3. Geometry of the representative volume elements (RVE) 


\begin{tabular}{|c|c|c|}
\hline RVE & Number of nodes & Number of elements \\
\hline 3x3-a & 64 & 96 \\
\hline $3 \times 3-b$ & 41 & 59 \\
\hline $3 \times 3-c$ & 47 & 69 \\
\hline $3 \times 3-p e r i o d i c$ & 38 & 54 \\
\hline $4 \times 4-a$ & 198 & 341 \\
\hline $4 \times 4-b$ & 63 & 97 \\
\hline $4 \times 4-c$ & 64 & 101 \\
\hline $4 \times 4-$ periodic & 59 & 91 \\
\hline $5 \times 5-a$ & 201 & 353 \\
\hline $5 \times 5-b$ & 91 & 148 \\
\hline $5 \times 5-c$ & 84 & 137 \\
\hline $5 \times 5-p e r i o d i c$ & 80 & 130 \\
\hline
\end{tabular}

Table 4. Number of nodes and number of triangular elements of the representative volume elements (RVE) 


\begin{tabular}{|c|c|c|c|}
\hline Joints & $\begin{array}{c}\text { Heterogeneous model } \\
\text { (\% of self-weight) }\end{array}$ & $\begin{array}{c}\text { Homogenous model } \\
\text { (\% of self-weight) }\end{array}$ & $\begin{array}{c}\text { Accuracy of } \\
\text { homogeneous model (\%) }\end{array}$ \\
\hline Weak mortar & 8.7 & 6.9 & 80 \\
\hline Strong mortar & 34.4 & 31.6 & 90 \\
\hline
\end{tabular}

Table 5. Comparison of limit loads from the heterogeneous model and homogeneous model. 